\title{
The organization of multidimensional selection on the basis of color and shape: An event-related brain potential study
}

\author{
H. G. O. M. SMID, A. JAKOB, and H.- J. HEINZE \\ Otto-von-Guericke University, Magdeburg, Germany
}

\begin{abstract}
In this paper, we examine whether color and shape, tied to a single object in space, (1) are identified and selected in series or in parallel, (2) are identified and selected in a dependent, self-terminating manner or in an independent and exhaustive manner, and (3) are conjoined by a feature integration process before or only after an initial stage of separate attribute analyses has finished. We measured response time and the selection negativity (SN) derived from event-related brain potentials when participants responded to a unique conjunction of color and shape in a go/no-go target detection task. The discriminability of the color and the shape of the conjunction was manipulated in three conditions. When color and shape were easy to discriminate, the SNs to color and shape started at the same time. When one attribute was less discriminable, the SN to that attribute started later, but not the SN to the complementary attribute. This suggests that color and shape are identified and selected in parallel. In all three discriminability conditions, the SNs to color and shape were initially independent but later interacted. This suggests that color and shape are initially selected independently and exhaustively, after which their conjunction is analyzed. The SN to local shape features started later than that to the conjunction of color and global shape features, which suggests that feature integration can start before the analyses of the separate attributes have finished.
\end{abstract}

A widely accepted model for multidimensional selection based on a conjunction of stimulus attributes proposes that such selection is accomplished in two contingent stages. In an initial stage, the attributes of a stimulus are processed separately and in parallel by specialized perceptual analyzers. In a second contingent stage, the outputs from the first stage are integrated to identify their conjunction. The results of the second stage are made available for central decision and motor-related processes. This model is supported by behavioral evidence (e.g., Hoffman, 1979; Treisman, 1993; Treisman \& Gelade, 1980) and evidence obtained with event-related brain potentials (ERPs; e.g., Hansen \& Hillyard, 1983; Harter \& Aine, 1984; Hillyard \& Münte, 1984; Smid, Böcker, Van Touw, Mulder, \& Brunia, 1996; Wijers, Mulder, Okita, \& Mulder, 1989; Woods \& Alain, 1993; Woods, Alho, \& Algazi, 1994). In the present study, we investigated multidimensional selection of conjunctions of color and shape to evaluate several aspects of the temporal and functional organization of the two-stage model.

Color and shape are separable stimulus attributes (e.g., Garner, 1974, 1987; Watanabe, 1988). The temporal and

The authors thank Lisa Fournier, Steve Hillyard, Art Kramer, Wolfgang Schwarz, and two anonymous reviewers for their helpful comments and suggestions concerning earlier versions of this article. The authors further thank Hermann Hinrichs, Michael Scholz, and HansJürgen Warmbold for their technical support. Correspondence should be addressed to H. G. O. M. Smid, Otto-von-Guericke University, Medical Faculty, Clinic for Neurophysiology, Leipziger Strasse 44, D-39120 Magdeburg, Germany (e-mail: henderikus.smid@medizin. uni-magdeburg.de). functional organization of the processing of stimuli with separable attributes can be described with analytic models (e.g., Egeth, 1966; Hawkins, 1969; Snodgrass \& Townsend, 1980; Townsend \& Ashby, 1983). Analytic models can vary on at least two orthogonal dimensions. Figure 1 illustrates some examples on these dimensions. One dimension describes whether multiple attributes are processed in series or in parallel. Figure $1 \mathrm{~A}$ illustrates a serial organization. In this case, the analyses are performed one after the other, and an analysis, $B$, can only start after its preceding analysis, $A$, has finished. The total time necessary to perform both analyses is the sum of their individual durations. If an experimental manipulation prolongs the duration of an earlier analysis $(A)$, the succeeding analyses $(B)$ must start and finish later. Figure 1B illustrates a parallel organization. In this case, the analyses start at the same time, and the total completion time is the completion time of the analysis taking longest. If an experimental manipulation prolongs a shorter analysis, total completion time increases only if the prolonged analysis becomes the longest analysis.

The second dimension describes whether multiattribute selection occurs in a self-terminating or in an exhaustive fashion. If multiattribute selection occurs in a selfterminating fashion, as illustrated in Figure 1C, processing of the stimulus is terminated whenever sufficient evidence has accrued, indicating that the value of one of the attributes is irrelevant for the task at hand. The left panel of Figure 1C illustrates the selection process when an attribute, $A$, can be identified faster than another attribute, $B$. In this case, as soon as $A$ is identified as having a non- 


\section{A Analyses arranged in series:}

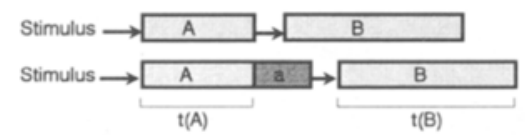

$t(A)+t(B)$

$t(A)+t(B)+a$

\section{B Analyses arranged in parallel:}

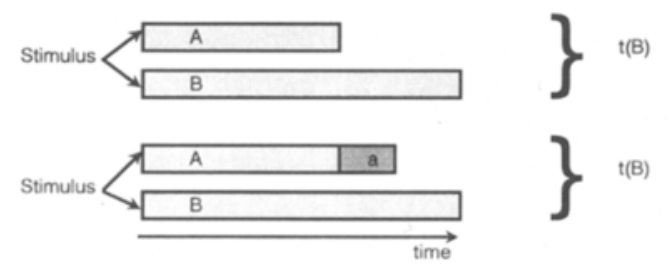

C Self-terminating selections:
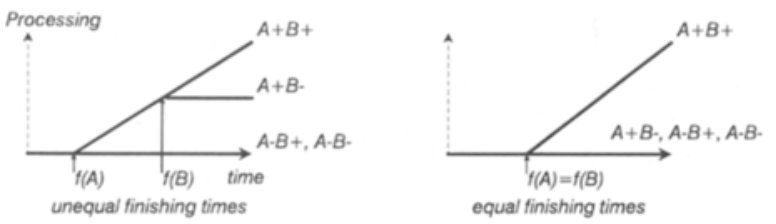

D Exhaustive selections:
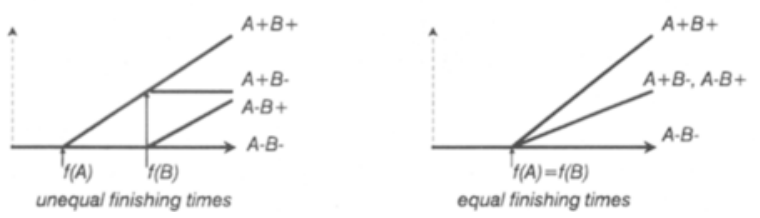

Figure 1. Serial/parallel and self-terminating/exhaustive processing in analytic multidimensional processing models. (A) A serial model in which an attribute $A$ is analyzed first. Only after the analysis of $A$ has finished can the analysis of attribute $B$ begin. The upper row shows that the time to complete both analyses is the sum of the durations of the analyses of $A, t(A)$ and $B, t(B)$. In the lower row, the analysis of $B$ starts later because analysis of $A$ is prolonged with time $a$ so that the completion time of both analyses becomes $t(A)+t(B)+a$. (B) A parallel model in which the analyses of $A$ and $B$ start at the same time but have different finishing times. In the upper example, the time to complete both analyses equals the time of the longest analysis, $t(B)$. The lower example shows that if analysis of $A$ is prolonged with $a$, and $t(A)+a$ is smaller than $t(B)$, the completion time of both analyses is still $t(B)$. (C) Self-terminating multiattribute selections, in which processing of a stimulus is terminated as soon as evidence is available indicating that the stimulus is irrelevant. The $\boldsymbol{x}$-axis represents time, and the $y$-axis represents amount of processing. The finishing times of the analyses of attributes $A$ and $B$ are denoted as $f(A)$ and $f(B)$. Attributes $A$ and $B$ have a relevant value $(+)$ or an irrelevant value $(-)$. The left figure illustrates selfterminating processing when attribute $A$ is available earlier than $B$. Note that if $A$ is irrelevant $(A-)$ selection on the basis of $B$ does not occur. The right figure illustrates self-terminating processing when $A$ and $B$ are available at the same time. Note that stimuli with one or more irrelevant attributes are not selected for further processing. (D) Exhaustive selections when $A$ is available earlier than $B$ (left illustration) and when $A$ and $B$ are available at the same time (right illustration). Note that stimuli with one irrelevant attribute value are selected for further processing. target value, processing of the stimulus is terminated and selection on the basis of attribute $B$ does not occur $(A-B+$ and $A-B-$ are not processed differently). Only when $A$ has a target value $(A+)$ does selection based on attribute $B$ occur ( $A+B+$ and $A+B-$ are differentially processed). Figure 1D illustrates exhaustive multiattribute selections. In this case, selection based on each attribute occurs, regardless of whether another attribute has a nontarget value or not. Thus, after attribute $A$ is identified as having a nontarget value, selection based on attribute $B$ would also occur $(A-B+$ and $A-B-$ are processed differently). In self-terminating models, multiattribute selection occurs in a dependent fashion in the sense that selection based on attribute $B$ depends on whether attribute $A$ has a target value. In exhaustive models, multiattribute selection occurs independently in the sense that selection based on attribute $B$ occurs regardless of whether or not $A$ has a target value.

In the present study, we focus on three questions regarding the identification and selection for further, taskdirected processing of the color and shape of a stimulus. First, are color and shape, presented in a single location of the visual field, identified and selected in series or in parallel? Second, are color and shape selected exhaustively and independently or in a self-terminating and dependent fashion. Third, does feature integration (i.e., conjunction analysis) of color and shape take place only after the separate attributes have been identified (i.e., the first and second stages of the two-stage model occur in series)? Or can feature integration start before identification of the separate attributes has finished? For example, Stage 2 may start as soon as the Stage 1 analyses of two easy discriminable attributes are finished and the analysis of a hard to discriminate attribute is still continuing.

At present, there is little and also conflicting evidence on these questions. Many behavioral findings suggest that color and shape are identified in parallel in different visual field locations. There is less evidence that they are identified in parallel in a single location, or independent of location - that is, at a level of functional parallellism instead of spatial parallellism (see Arguin \& Cavanagh, 1988). Several behavioral studies support functional parallellism of color and shape identification (e.g., Arguin \& Cavanagh, 1988; Biederman \& Checkosky, 1970; Ellis \& Chase, 1971; Hawkins, 1969; McClean, Broadbent, \& Broadbent, 1982; Saraga \& Shallice, 1973; van der Heijden, La Heij, Phaf, Buijs, \& van Vliet, 1988). Other studies, however, have reported evidence supporting serial identification of color and shape (e.g., Egeth, 1966; Grill, 1971). Most of the existing physiological evidence, based on intracranial ERPs, concerns passive parallel processing of elementary stimulus parameters (Desimone \& Ungerleider, 1989). Most ERP studies, and reviews of these studies, which evaluate the issue of dependent versus independent attribute processing, assume that, in general, multiattribute processing occurs in a self-terminating 
fashion (e.g., Hillyard \& Münte, 1984; Hillyard \& Picton, 1987; Näätänen, 1992). This contrasts with findings obtained with the lateralized readiness potential by Smid, Mulder, Mulder, and Brands (1992), who showed that color and shape can be independently processed up to the level of selective response activation. It also contrasts with findings obtained by Kenemans, Kok, and Smulders (1993), who showed that spatial frequency and orientation are identified and selected independently. Mordkoff and Yantis (1993) reported behavioral evidence suggesting that color and shape, tied to a single object, may be processed independently in an early stage, after which their processing interacts according to a coactivation model. In the auditory modality, the evidence suggests that feature integration can begin before individual attribute analyses have finished (Woods \& Alain, 1993). Such evidence is lacking for the visual modality.

\section{An ERP Measure of Differential Task-Directed Processing: The Selection Negativity}

We studied these issues with a measure derived from ERPs. Previous studies using the ERP method to investigate selective attention have shown that if color, spatial frequency, and orientation serve as selection cues (e.g., "attend the red stimuli and ignore the blue stimuli"), specific ERP responses can be observed that are related to the selective, task-directed, processing of stimuli with the cued attribute (for reviews and critical commentaries, see Harter \& Aine, 1984, 1986; Hillyard \& Mangun, 1986; Hillyard \& Picton, 1987; Mangun \& Hillyard, 1995; Näätänen, 1992; Rugg, 1991; and Wijers, 1989). In a recent study, Smid et al. (1996) found that, when shape is a selection cue, it elicits a similar ERP response. This response, which typically starts between 120 and $320 \mathrm{msec}$ after stimulus presentation, is maximal over occipital scalp sites, is specific for the visual modality, and has a negative polarity, is called selection negativity (SN; Harter \& Aine, 1984). It concerns a relatively slow endogenous negative shift that overlaps ERP components related to passive information processing.

The SN is a difference potential, derived by subtracting ERPs elicited by stimuli with a nontarget (irrelevant) value on a dimension (e.g., blue) from ERPs elicited by stimuli with the target (relevant, cued) value on that dimension (e.g., red). To obtain an SN specifically related to the task relevance of the dimensional value and to eliminate ERP differences caused by physical differences between dimensional values, each physical value serves as the relevant value in some trial blocks and as the irrelevant value in others. The ERPs to the relevant and irrelevant values are then averaged across the different physical values. If this subtraction results in a significant amplitude difference, it can be inferred that stimuli with the target value were differentially processed as a function of attention relative to stimuli with the nontarget value (Näätänen, 1992; Rugg, 1991). The onset latency of the SN can be assumed to index the upper limit of the time at which the cognitive system has discriminated the target value of the attribute from the nontarget value of the attribute (Näätänen, 1992; Rugg, 1991). Therefore, at that time, the cognitive system logically must have identified the attribute to at least the extent that it can discriminate target from nontarget attribute values. Thus, if relevant and irrelevant attribute values produce an ERP difference identifiable as SN, it can be inferred that (1) the attribute was identified and (2) the stimuli with the relevant value received more task-directed processing than did the stimuli with the irrelevant value. ${ }^{1}$

When stimuli have to be processed on the basis of multiple attributes, the SN related to the selective processing of each of the attributes can be derived. This is done by recording occipital ERPs to stimuli that consist of two attributes, $A$ and $B$, presented one at a time. Each of these attributes can have one of two values that vary orthogonally. Thus, there are four possible combinations of $A$ and $B$ values. The task of the participants is to attend to one of these combinations and to determine whether or not it has a third, difficult-to-discriminate attribute. If it has the third attribute, the stimulus is a target, and a simple detection response must be made. The participants should not respond to all other stimuli that are presented (the nontargets). For example, Hillyard and Münte (1984) investigated multidimensional selection of the location $(A)$ and the color $(B)$ of bars and used a small variation in bar length as the third attribute. The third attribute is made the least discriminable to ensure that attributes $A$ and $B$ are identified first on trials on which no response is required. In this way, the SNs related to the selective processing of attributes $A$ and $B$ can be examined without being contaminated by ERP effects related to producing a response. We label the relevant attribute values with a plus $(+)$ sign and the irrelevant values with a minus $(-)$ sign. Four possible nontarget stimuli of main experimental interest are presented in the task: $A+B+$ stimuli share both the attribute values of $A$ and $B$ with the target; $A+B-$ stimuli only share the value of attribute $A$ with the target; $A-B+$ stimuli only share the value of attribute $B$ with the target; and $A-B-$ stimuli share none of the attribute values with the target.

The time course of the selection of attributes $A$ and $B$ can be inferred from the time points at which the occipital ERPs to these stimuli start to differ as a function of $A$ and $B$ relevance. The onset latency of the difference between the ERPs to $A^{+}$and $A-$ stimuli marks the time at which identification and selection on the basis of attribute $A$ has occurred. Likewise, the onset latency of the difference between the ERPs to $B+$ and $B$ - stimuli is related to the identification and selection of attribute $B$. Thus, if the $A+$ and $A$ - ERPs start to differ earlier than the $B+$ and $B$ - ERPs, we would infer that selection of $A$ occurred earlier than selection of $B$. We first describe the logic to infer whether $A$ and $B$ are selected in a self-terminating or exhaustive fashion and then describe the logic to infer whether $A$ and $B$ are identified and selected in series or in parallel. 
Table 1

Predictions Regarding SN Amplitude Effects as a Function of Stimulus Relevance Derived From Self-Terminating and Exhaustive Analytic Models and Holistic Models of Multidimensional Stimulus Selection

\begin{tabular}{cccccccc}
\hline & \multicolumn{2}{c}{ Self-Terminating } & Exhaustive & \multirow{2}{*}{ Holistic } & \multicolumn{3}{c}{ Results } \\
\cline { 2 - 3 } \cline { 6 - 7 } Prediction & Model 1 & Model 2 & Model* & Model & CESE & CHSE & CESH \\
\hline (1) $[\operatorname{ERP}(A+B+)-\operatorname{ERP}(A-B+)]$ & $>0$ & $>0$ & $>0$ & $>0$ & $>0$ & $>0$ & $>0$ \\
(2) $[\operatorname{ERP}(A+B+)-\operatorname{ERP}(A+B-)]$ & $>0$ & $>0$ & $>0$ & $>0$ & $>0$ & $>0$ & $>0$ \\
(3) $[\operatorname{ERP}(A+B-)-\operatorname{ERP}(A-B-)]$ & $>0$ & $=0$ & $>0$ & $=0$ & $>0$ & $>0$ & $>0$ \\
(4) $[\operatorname{ERP}(A-B+)-\operatorname{ERP}(A-B-)]$ & $=0$ & $=0$ & $>0$ & $=0$ & $>0$ & $>0$ & $=0$
\end{tabular}

*Additional Predictions of the Exhaustive Model (Predictions $1-4$ all $>0$ ):

(5) $[\operatorname{ERP}(A+B+)-\operatorname{ERP}(A-B+)] \quad=[\operatorname{ERP}(A+B-)-\operatorname{ERP}(A-B-)]>0$;

(6) $[\operatorname{ERP}(A+B+)-\operatorname{ERP}(A+B-)]=[\operatorname{ERP}(A-B+)-\operatorname{ERP}(A-B-)]>0$;

Later, if Processing Becomes Selective for the $A+B+$ Conjunction:

(7) $[\operatorname{ERP}(A+B+)-\operatorname{ERP}(A-B+)]>[\operatorname{ERP}(A+B-)-\operatorname{ERP}(A-B-)]>0$;

(8) $[\operatorname{ERP}(A+B+)-\operatorname{ERP}(A+B-)]>[\operatorname{ERP}(A-B+)-\operatorname{ERP}(A-B-)]>0$.

Note-For self-terminating models, a distinction is made between the situation in which the identification of $A$ finishes before identification of $B$ (self-terminating Model 1) and the situation in which identifications of $A$ and $B$ finish at the same time (self-terminating Model 2). Note that the self-terminating model makes the same predictions as the holistic model when identifications of $A$ and $B$ finish at the same time (Figure 1 gives a graphical illustration of these predictions). The columns under Results give the findings obtained in the three discriminability conditions (CESE, CHSE, and CESH). The lower part of the table (Predictions 5-8) shows how independent processing of attributes $A$ and $B$ can be discriminated from selective processing of the $A+B+$ conjunction when Predictions $1-4$ of the exhaustive model are confirmed.

\section{Self-Terminating Versus Exhaustive Processing}

Whether selection of $A$ and $B$ occurs in a self-terminating and dependent manner or occurs exhaustively and independently is inferred from an analysis originally proposed by Hansen and Hillyard (1983) and was later successfully applied by other researchers (Harter \& Aine, 1984; Hillyard \& Münte, 1984; Kenemans et al., 1993; Wijers, Lamain, Slopsema, Mulder, \& Mulder, 1989; Wijers, Mulder, et al., 1989; Woods et al., 1994). In this analysis, each of the effects of $A$ and $B$ relevance is evaluated as a function of the level of relevance of the complementary attribute. The ERPs recorded to each stimulus type are subtracted in four different combinations, in which one attribute is varied and the other attribute remains constant. The difference wave $[\operatorname{ERP}(A+B+)$ - $\operatorname{ERP}(A-B+)]$ represents the SN related to the selection of attribute $A$ for the stimuli that have a relevant $B$ value. The $[\operatorname{ERP}(A+B-)-\operatorname{ERP}(A-B-)]$ difference represents the SN related to the selection of attribute $A$ for the stimuli that have an irrelevant $B$ value. Similarly, the difference wave $[\operatorname{ERP}(A+B+)-\operatorname{ERP}(A+B-)]$ represents the SN related to the selection of attribute $B$ for the stimuli that have a relevant $A$ value. The $[\operatorname{ERP}(A-B+)-$ $\operatorname{ERP}(A-B-)]$ difference represents the $\mathrm{SN}$ related to the selection of attribute $B$ for the stimuli that have an irrelevant $A$ value. Table 1 lists the predictions derived from self-terminating, exhaustive, and holistic models for each of these four difference waves. Figures $1 \mathrm{C}$ and 1D show graphical representations of the logic underlying these predictions.

The self-terminating model makes different predictions, depending on whether attributes $A$ and $B$ are identified at the same time or whether one is identified earlier than the other. If attribute $A$ is identified earlier than $B$ is, the ERP differences related to the selection of at- tribute $A$ would occur first, both if $B$ was relevant and if $B$ was not relevant. In Table 1, this is represented by Prediction 1, $[\operatorname{ERP}(A+B+)-\operatorname{ERP}(A-B+)]>0$, and by Prediction 3, $[\operatorname{ERP}(A+B-)-\operatorname{ERP}(A-B-)]>0$. Furthermore, $B$ would be selected if $A$ had a relevant value but not if $A$ had an irrelevant value, because processing would be terminated as soon as information was available indicating that $A$ was irrelevant. This is represented by Prediction 2, $[\operatorname{ERP}(A+B+)-\operatorname{ERP}(A+B-)]>0$, and by Prediction 4, $[\operatorname{ERP}(A-B+)-\operatorname{ERP}(A-B-)]=0$, respectively. Thus, selection of $B$ would depend hierarchically on selection of $A$. If the identities of attributes $A$ and $B$ become available at the same time, processing would be terminated if $A$ or $B$ had an irrelevant value. In this case, the self-terminating model predicts (1) $[\operatorname{ERP}(A+B+)-$ $\operatorname{ERP}(A-B+)]>0$ and $(2)[\operatorname{ERP}(A+B+)-\operatorname{ERP}(A+B-)]$ $>0$, but (3) $[\operatorname{ERP}(A+B-)-\operatorname{ERP}(A-B-)]=0$ and (4) $[\operatorname{ERP}(A-B+)-\operatorname{ERP}(A-B-)]=0$.

If selection occurs exhaustively, all the relevant and irrelevant attribute values (e.g., $B+$ and $B-$ ) lead to differential task-directed processing, even if a complementary attribute has an irrelevant value $(A-)$. Thus, all four ERP differences should be larger than zero after both attributes are identified (Predictions $1-4$ of the exhaustive model in Table 1). Because all four differences are present in the ERPs, it is possible to determine when processing becomes selective for the relevant conjunction of $A$ and $B$ (i.e., for $A+B+$ stimuli). We refer to this time point as the time at which feature integration of $A$ and $B$ must have started. This analysis involves testing four additional predictions (Predictions 5-8 in Table 1) at different time points. Initially, if the attributes are processed independently, Prediction 5, $[\operatorname{ERP}(A+B+)-\operatorname{ERP}(A-B+)]=$ $[\operatorname{ERP}(A+B-)-\operatorname{ERP}(A-B-)]>0$, and Prediction 6, $[\operatorname{ERP}(A+B+)-\operatorname{ERP}(A+B-)]=[\operatorname{ERP}(A-B+)-$ 
$\operatorname{ERP}(A-B-)]>0$, must hold. Thus, the difference between the $A+B+$ ERP on the one hand and the $A+B-$ and $A-B+$ ERPs on the other hand should be equal to the difference between the $A+B-$ and $A-B+$ ERPs on the one hand and the $A-B-$ ERP on the other (see Figure 1D). This means that the amount of SN produced by a stimulus must be proportional to the number of relevant attribute values of a stimulus. In statistical terms, this means that there are additive main effects, but no interaction, of $A$ relevance and $B$ relevance. If, at some later time point, processing becomes selective for the relevant $A+B+$ conjunction, Prediction 7, $[\operatorname{ERP}(A+B+)-\operatorname{ERP}(A-B+)]>$ $[\operatorname{ERP}(A+B-)-\operatorname{ERP}(A-B-)]>0$, and Prediction 8, $[\operatorname{ERP}(A+B+)-\operatorname{ERP}(A+B-)]>[\operatorname{ERP}(A-B+)-$ $\operatorname{ERP}(A-B-)]>0$, must hold. That is, the difference between the $A+B+$ ERP on the one hand and the $A+B-$ and $A-B+$ ERPs on the other hand should be larger than the difference between the $A+B-$ and $A-B+$ ERPs on the one hand and the $A-B-$ ERP on the other (see Figure 1D). ${ }^{2}$ This means that the amount of $\mathrm{SN}$ elicited by an $\mathrm{A}+\mathrm{B}+$ stimulus is disproportionately large. In statistical terms, this means that there are main effects and an interaction of $A$ and $B$ relevance. The onset latency of this conjunctionspecific processing indicates that the $A+B+$ stimulus must have been identified as the relevant conjunction.

The holistic model predicts the same pattern of differences as the self-terminating model with equal identification times of attributes $A$ and $B$. That is, (1) $[\operatorname{ERP}(A+B+)$ $-\operatorname{ERP}(A-B+)]$ and (2) $[\operatorname{ERP}(A+B+)-\operatorname{ERP}(A+B-)]$ differences are larger than zero, but (3) $[\operatorname{ERP}(A+B-)-$ $\operatorname{ERP}(A-B-)]$ and (4) $[\operatorname{ERP}(A-B+)-\operatorname{ERP}(A-B-)]$ differences are equal to zero. This pattern means that the ERPs to $A+B-, A-B+$, and $A-B-$ stimuli were indistinguishable, and that only the ERP to $A+B+$ stimuli was different. The self-terminating model with equal identification times differs from the holistic model in that the former allocates task-directed processing on the basis of separate representations of color and shape, whereas the latter allocates processing on the basis of a single representation of the conjunction of color and shape.

\section{Serial Versus Parallel Processing}

Whether identification and selection of multiple attributes occur in series or in parallel can be inferred by manipulating the time needed to identify the attributes and observing the onset latencies of the SNs related to the selection of the two attributes. We manipulated the attribute identification times by varying the relative discriminability of the color and shape attributes in three different conditions. In the color-easy/shape-easy (CESE) condition, the color and the shape of the stimuli were both easy to discriminate. In the color-hard/shape-easy (CHSE) condition, the color was hard to discriminate and the shape was easy. In the color-easy/shape-hard (CESH) condition, the color was easy and the shape was hard to discriminate.

In the CESE condition, we could obtain two different patterns of SN results, each consistent with two hypotheses, that could be distinguished as a function of attribute discriminability. First, in the CESE condition, the onset latencies of the SNs related to color and shape selection might differ. This is consistent with the hypothesis that color and shape were identified and selected one after the other (i.e., in series). It is also consistent, however, with the hypothesis that color and shape were identified and selected in parallel and that the time needed for their identification differed. These hypotheses can be discriminated with the aid of the results from the CHSE and CESH conditions. If, for example, in the CESE condition, the SN related to the selection of color would start before the $\mathrm{SN}$ related to shape selection and the color and shape selections took place in a serial fashion, then making the color hard to discriminate should delay not only the SN related to color selection but also the $\mathrm{SN}$ related to shape selection (see Figure 1A). If the identification of color and shape occurred in parallel, with identification of shape terminating later than that of color, then making the color hard to discriminate should not delay the onset of the $\mathrm{SN}$ related to shape selection (Figure 1B).

Second, in the CESE condition, we could obtain equal $\mathrm{SN}$ onset latencies related to color and shape selection. This seems most compatible with the hypothesis that the analyses of color and shape were performed in parallel and took an equal amount of time. However, the rival hypothesis is that the equal $\mathrm{SN}$ onsets were the result of averaging together two types of serial processing, each performed on a different group of trials. The $\mathrm{SN}$ is derived by subtracting two ERP averages, each obtained from a large number of trials. Suppose that, on about half of these trials, color was selected before shape, and, on the other half of the trials, shape was selected before color. Suppose further that on color-first trials, color was selected at the same time as shape on shape-first trials. After averaging color-first and shape-first trials together, the SN effects of color and shape relevance would have equal onsets despite the serial nature of selections on individual trials.

Because the $\mathrm{SN}$ is not identifiable on single trials, but only as a difference in the averages of single subjects, this can be an important problem when using the $\mathrm{SN}$ (see, e.g., Rugg, 1991). Fortunately, the trial-mixture serial model makes a falsifiable prediction about the amplitude and duration of the average $\mathrm{SN}$. This prediction is that, on the color-first trials, an early SN related to color selection would be followed by an $\mathrm{SN}$ related to shape selection. On shape-first trials, an early shape-related SN should be followed by a color-related SN. By averaging these two trial types, the early color-related $\mathrm{SN}$ on colorfirst trials would be averaged with the later color-related SN on shape-first trials, so that those parts of the SNs that do not temporally overlap on color-first and shapefirst trials are drastically reduced in amplitude. In the same vein, the early shape-related $\mathrm{SN}$ on shape-first trials would be averaged with the later shape-related SN on color-first trials. As a result, these SNs, averaged across both types of trials, should have a much smaller amplitude and a much longer duration than the amplitudes and durations of the SNs obtained on shape-first trials and color-first trials separately. The results from the CHSE and CESH conditions can be used to evaluate this prediction. 
Independent of whether identification occurred in series or in parallel, the difference in discriminability would ensure that, on the majority of the trials in the CHSE condition, shape identification would be ready first, and, on the majority of the trials in the CESH condition, color identification would be ready first. On this logic, the SNs obtained in the CHSE condition can be used as estimates of the SNs in shape-first trials, and the SNs obtained in the CESH condition can be used as estimates of the SNs in color-first trials. Thus, if we would obtain equal SN onsets related to color and shape selection in the CESE condition, we could infer that color and shape were analyzed in parallel, unless the shape-related SN in the CHSE condition and the color-related SN in the CESH condition had a larger amplitude and shorter duration than those of the shape- and color-related SNs in the CESE condition.

We used a multidimensional selective attention task, in which participants made a simple detection response to a conjunction of color, global shape, and local shape. The shapes in this task were designed such that variation in the position of a local shape element (e.g., a gap in a circle) did not alter the nature of the global shape (e.g., a circle). In this way, the shapes conform to what Pomerantz (1983) termed Type $P$ stimuli (P for position) - that is, stimuli that have separable global and local shape attributes that can be selectively attended. The difficulty of global and local shape identification was manipulated, so that local shape identification took longer than did color and global shape identification in the CESE condition. In the CHSE condition, the colors were made hard to discriminate, whereas the global and local shapes were equal to those in the CESE condition. In the CESH condition, identification of the global shape was made hard, whereas identification of the local shape required the same local shape discrimination as in the CESE condition (see Method section below for details). The local shape therefore played the role of the third attribute, explained earlier, in all three conditions. Thus, the participants had to attend a relevant-color-global-shape combination to determine the presence of a local shape requiring a buttonpress. In contrast to previous ERP studies on multidimensional selection, in the present study, the local shape attribute was explicitly used because its relevant and irrelevant values produce ERP differences that are identifiable as SNs and that could not be attributed to response-related processing. Thus, by recording ERPs to the stimuli in this task, we could derive the $\mathrm{SN}$ related to the selection of each of the three attributes. We use the onset latency of the interaction of color relevance and global shape relevance to infer the time the color/global shape conjunction had been identified (i.e., the time feature integration of the color and the global shape must have started). With regard to the question of whether or not feature integration can start before the separate attribute analyses are ready, we observe (1) whether the $\mathrm{SN}$ related to local shape selection occurs before or after the onset of conjunction specific processing and (2) how the discriminability manipulation affects the onsets of local shape selection and conjunction-specific processing
ERPs. If the local shape SN starts later than conjunctionspecific processing of color and global shape and if decreasing the discriminability of color or global shape delays conjunction-specific processing of color and global shape but not local shape analysis, we could infer that feature integration can start before all separate attributes had been identified. ${ }^{3}$

\section{METHOD}

\section{Participants}

Eighteen right-handed students of the University of Magdeburg ( 8 females, 10 males; mean age $=24.5$ years, age range $=19-29$ years), with normal or corrected vision) participated in the experiment.

\section{Stimuli}

Nonalphanumeric shapes were designed on the basis of two easily discriminable global shape configurations: closure and vertical parallel lines (e.g., Kimchi, 1992; Treisman \& Paterson, 1984). Each configuration had four variants that differed only in a small shape segment. These variants could have one of four colors: red, light red, blue, and light blue. Figure 2 shows the stimuli that resulted after testing and adjusting the initial stimuli in a preliminary experiment designed to make color and shape discriminability relatively equal. ${ }^{4}$

The red and blue colors consisted of color mixture values in the VGA 256 color pallette of the IBM stimulus-presentation PC: red = $63 \mathrm{red}, 0$ green, 0 blue; blue $=0$ red, 0 green, 63 blue. The light-

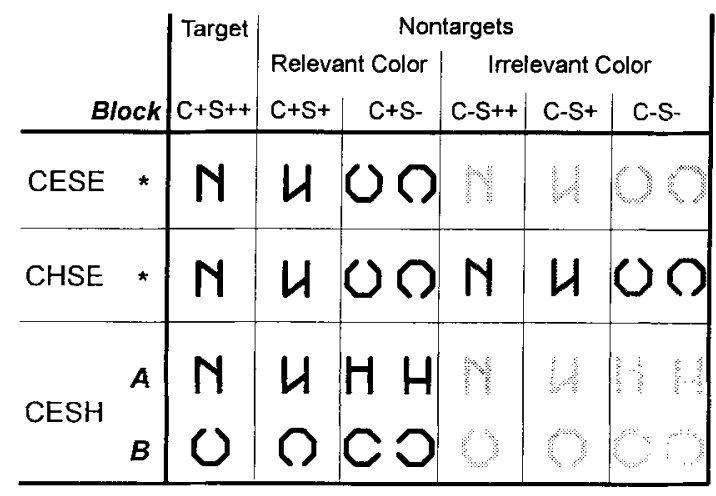

Figure 2. An example of the $S-R$ mappings used in the ERP experiment. CESE denotes the color-easy/shape-easy condition; CHSE denotes the color-hard/shape-easy condition; and CESH denotes the color-easy/shape-hard condition. The target requiring a response is in the $\boldsymbol{C}+\boldsymbol{S}++$ column (the color attribute and the global and local shape attributes are relevant). The nontargets, which required no response, could share the color and the global shape with a target (relevant color, $C+S+$ column), only the color (relevant color, $C+S-$ column), global and local shape but not the color (irrelevant color, $C-S++$ column), only the global shape (irrelevant color, $C-S+$ column), or no attribute with the target (irrelevant color, $C-S-$ column). The $S$ - stimuli $(C+S-$ and $C-S-)$ consisted of two alternatives (to balance this category with the $S+$ categories). Only one of each of these stimulus shapes was presented during a trial. Differences in color are shown as differences in hatching texture. For example, if the completely filled shapes represent blue (all $C+$ stimuli), the least filled shapes represent red ( $C-$ in CESE and $C E S H$ ), and the intermediate filled shapes represent light blue (in CHSE). Two examples are shown of the mapping in the CESH condition $(A$ and $B$ ). The star (*) in CESE and CHSE indicates that in these conditions there were also multiple $S-R$ mappings. 
red and light-blue colors consisted of other mixtures: light red $=$ 63 red, 22 green, 0 blue; light blue $=0$ red, 25 green, 63 blue. The stimuli were presented on a PC-controlled video monitor on a black background at a viewing distance of $100 \mathrm{~cm}$. At this distance, they subtended $2.5^{\circ} \times 2.5^{\circ}$ of visual angle.

The stimuli shown in Figure 2 were presented, one at a time, in a fixated central location of a video screen. They varied on the dimensions color, global shape, and local shape. One conjunction of color (e.g., blue), global shape (e.g., a pair of vertical parallel lines), and a particular shape element (e.g., a diagonal line between the parallel lines), served as the target for a simple keypress. We use acronyms to indicate the various stimulus types. Thus, $C$ stands for color, $S$ stands for shape, "+" indicates an attribute that is shared with the target, and "-" indicates an attribute that differs from the target. Targets are denoted as $C+S++$ stimuli because the color $(C+)$ and two shape attributes $(S++)$ identify them as targets (the leftmost column in Figure 2). Nontargets, requiring no response, could have the same color as a target $(C+)$, but only one relevant shape attribute $(S+)$, the global shape. These stimuli had an irrelevant local shape element $(C+S+$ stimuli; e.g., blue parallel lines with a differently oriented diagonal in the wrong location). Other nontargets shared either only the color $(C+S-$; e.g., a blue circular shape) or only the global shape with a target $(C-S+$; e.g., red parallel lines); others shared none of these attributes with the target ( $C-S-$; e.g., a red circular shape). One type of nontarget shared both shape attributes with the target but not its color $(C-S++$; e.g., red parallel lines with the same diagonal as the $C+S++$ target). Thus, $S++$ stands for stimuli that shared the global shape and the local shape with the target, $S+$ stands for stimuli that shared only the global shape with the target, and $S$ - stands for stimuli that shared no shape attributes with the target.

\section{Procedure}

The participants were seated in a reclining chair in a sound- and light-attenuated chamber. The experiment was run in one session (approximately $3 \mathrm{~h}$ ) on a single day. The session consisted of 20 blocks, each consisting of a randomized sequence of 240 stimulus presentations, divided over three discriminability conditions ( 4 blocks CESE, 8 blocks CHSE, and 8 blocks CESH, see above). The participants performed an equal number of trials with each type of discrimination (for colors, 4 blocks red vs. blue, 4 blocks light red vs. red, 4 blocks light blue vs. blue; for shapes, 4 blocks circular vs. vertical parallel, 4 blocks one circular variant vs. other circular variants, 4 blocks one vertical-parallel variant vs. other vertical-parallel variants). The probability of presentation of each of the eight possible stimuli (see Figure 2) was equal within each block (.125). Of all stimuli, $25 \%$ had a relevant color and a relevant global shape $(C+S+$ plus $C+S++), 25 \%$ had a relevant color and an irrelevant global shape $(C+S-), 25 \%$ had an irrelevant color and a relevant global shape $(C-S+$ plus $C-S++)$, and $25 \%$ had both an irrelevant color and an irrelevant global shape $(C-S-)$. Luminance and contrast of the stimuli were equal in the three discriminability conditions. In each block, a new combination of color and shape served as the target stimulus. This was done by letting each aiternative stimulus color and stimulus shape serve as a selection cue once in each discriminability condition. This counterbalanced the effects of physical stimulus differences. The order of the discriminability conditions and that of the different targets that the participants had to detect within discriminability conditions were counterbalanced between participants.

A block of trials began with a visual fixation aid (a small dot) in the center of the screen that remained visible during the entire block. The participants were told to fixate on the dot and to make as few eye movements and eye blinks as possible. The stimuli were presented for $100 \mathrm{msec}$. The interstimulus interval, measured from stimulus onset to stimulus onset, varied randomly between 700 and $1,100 \mathrm{msec}$.
The experiment started with an instruction. The participants were shown the to-be-detected target conjunction for the upcoming block. They were told that this conjunction would occur randomly in a stream of other color-shape combinations. They were further told that it was their task to press a button with the right hand only when the target was presented. This reaction had to be as quick as possible but with high accuracy. Next, the participants performed one or more training blocks of 80 trials each. Prior to the first experimental block, the participants performed at least three 80 -trial practice blocks to stabilize performance. When each new conjunction target was presented, the participants practiced the task until performance had stabilized prior to performing the experimental blocks. The next experimental block was then presented.

\section{Recording and Analysis}

The ERPs were recorded from the scalp using tin electrodes mounted in an electro cap (Electro-Cap International) and located at 29 sites according to the International 10-20 System: The standard sites included F7, F8, F3, F4, Fz, Cz, P3, P4, Pz, T3, T4, T5, $\mathrm{T} 6, \mathrm{O} 1, \mathrm{O} 2, \mathrm{Oz}, \mathrm{IN} 1, \mathrm{IN} 2$, and INz; the nonstandard sites included $\mathrm{FCl}$ (halfway between $\mathrm{Cz}$ and $\mathrm{F} 3$ ), C3' ( $1 \mathrm{~cm}$ anterior to $\mathrm{C} 3$ ), $\mathrm{CP} 1$ (halfway between $\mathrm{Pz}$ and $\mathrm{C} 3)$, PO1 $(0.5 \mathrm{~cm}$ lateral to the halfway point between $\mathrm{Pz}$ and $\mathrm{O} 1$ ), and $\mathrm{TO} 1$ (halfway between $\mathrm{Ol}$ and the midpoint of a line between $\mathrm{P} 3$ and $\mathrm{T} 5$ ), mirrored over the right hemisphere by $\mathrm{FC} 2, \mathrm{C}^{\prime}$, $, \mathrm{CP} 2, \mathrm{PO} 2$, and $\mathrm{TO} 2$. All these electrodes were referenced to the left earlobe. Eye blinks and eye movements were monitored with electrodes at both outer canthi of the eyes (horizontal electrooculogram, EOG) and below the right eye (vertical EOG).

The electrophysiological signals were filtered with a band-pass of $0.01-70 \mathrm{~Hz}$ (half-amplitude cutoffs) and digitized at a rate of $250 \mathrm{~Hz}$. Automated artifact rejection was performed off line to eliminate data epochs contaminated by blinks, saccades, excessive muscle activity (with a criterion of $50 \mu \mathrm{V}$ ), and amplifier saturation. The EEG signal was averaged, for each stimulus type in each condition, over epochs of $1,080 \mathrm{msec}$, starting $100 \mathrm{msec}$ before onset of the stimulus and ending $980 \mathrm{msec}$ poststimulus. This was done for each subject separately, and these averages were then used for statistical analyses (multivariate analyses of variance, MANOVAs).

Before the statistical analyses, the 0 - to $400-\mathrm{msec}$ poststimulus interval of the averages was divided into 50 epochs of $8 \mathrm{msec}$, averaging the amplitudes of each sequential pair of 4-msec samples in this interval and correcting for differences in the 100 -msec prestimulus baseline. This was done with the average of each stimulus type for each subject in order to decrease the number of epochs to be tested without loosing much temporal resolution. Next, the effects of stimulus relevance were evaluated to determine whether they were identifiable as $\mathrm{SN}$ effects. The a priori criteria for identifying a relevance effect as an $\mathrm{SN}$ effect were that the effect should (1) be maximal over occipital cortex and (2) start between 120 and $300 \mathrm{msec}$ after stimulus presentation. Visual inspection of the leftear referenced averages at the various electrodes clearly showed that the earliest relevance effects were maximal at the TO1 and TO2 electrodes (see Figure 3 ). This was confirmed by computing equipotential maps with BESA software (Scherg, 1992) on the grand averages, using common average reference. In all three discriminability conditions, the difference ERPs were maximal bilaterally in the areas of the TO1 and TO2 electrode sites, both when referenced to the left ear and when referenced to common average.

Finally, the amplitudes of the averaged ERPs to the various stimulus types were subjected to MANOVAs by means of planned comparisons according to a repeated measures within-subjects design. These comparisons concerned the difference between the ERPs to the following stimulus classes, each representing an effect of attribute relevance: $(C+)-(C-)$, the main effect of color relevance; $(S+)-(S-)$, the main effect of shape relevance; $(C+S+)-(C-S+)$ the effect of color relevance if the global shape is relevant; $(C+S-)$ $-(C-S-)$, the effect of color relevance if the global shape is ir- 
relevant; $(C+S+)-(C+S-)$, the effect of global shape relevance if the color is relevant; $(C-S+)-(C-S-)$, the effect of global shape relevance if the color is irrelevant; $(C+S++)-(C+S+)$, the effect of local shape relevance if the color and global shape are constant and relevant; and $(C-S++)-(C-S+)$, the effect of local shape relevance if the color is irrelevant and the global shape is relevant, see above.

These tests were carried out in order to determine, in each discriminability condition, (1) whether there was a significant difference between the averaged ERPs (i.e., whether an $\mathrm{SN}$ with significant amplitude was present), (2) the onset of that SN, and (3) the offset of the $\mathrm{SN}$ (in order to derive its duration). To reach these goals, we used statistical procedures that are standard in ERP research. Because ERPs on single trials contain much random noise, they must first be averaged to reduce the noise and to obtain identifiable ERPs. Next, the onset of an ERP difference is determined across participants. We did this by testing the fifty 8 -msec epochs in the 400-msec interval after stimulus presentation across participants. We distinguished two time ranges for two different purposes. In the 120- to 320 -msec prespecified interval (see introduction), we tested the null hypothesis that there were no effects of relevance. This hypothesis was rejected when a consecutive series of at least 3 epochs (representing a 24-msec interval) with $p$ values below .05 was found. The first of these epochs was taken as the onset latency of a significant $\mathrm{SN}$. The criterion of finding at least 3 epochs with $p$ values below .05 is a reasonable correction for performing many pairwise comparisons (interested readers may want to consult Guthrie \& Buchwald, 1991, for this procedure). In the initial 0- to $120-\mathrm{msec}$ interval, we tested the null hypothesis that there were effects of relevance at, according to the extant literature, unreasonably early time points, using the 3 -epoch criterion. This gave us an indication of the probability of making Type I errors. As will be shown in the Results section, there was not a single occurrence of a significant difference with a duration of 3 epochs in any condition for any pairwise comparison in this interval. This suggests that the occurrence of Type I errors was minimal and that the 3 -epoch restriction was a reasonable one.

The 3-epoch criterion was also used in our inferences about differences in SN onset. Because with ERPs it is not possible to obtain a statistical onset of a difference potential for each participant and condition, a direct statistical test on onsets across participants is not possible. Instead, the first time point (i.e., epoch) at which a statistically significant difference is found in one condition must be compared with the first time point at which a significant difference is found in another condition. These time points represent statistically "hard" points in time that separate series of epochs in which a difference was not present and series of epochs in which a difference was present. Since Type II and Type I errors can shift these time points over one or two epochs, a correction for such errors must be made. We used the same correction as that used for deciding whether a significant difference in amplitude was present. A meaningful difference in onset had to be larger than 3 epochs (i.e., $24 \mathrm{msec}$ ). Thus, a difference smaller than $24 \mathrm{msec}$ was taken as no difference, and a difference $24 \mathrm{msec}$ or larger was taken as a meaningful difference. In addition to this correction, we present the statistical results graphically in terms of alpha values as a function of time. This enables the reader to apply the most conservative correction on 50 repeated tests, which is to divide the adopted alpha level (.05) by the number (50) of repeated tests (Bonferroni). This correction requires a $p<.001$ level of significance in the present experiment. When applying this correction, however, be aware that it seriously compromises the power of the tests (Guthrie \& Buchwald, 1991). That is, meaningful effects repeated in, say, 5 consecutive epochs, which all reach an alpha level of $p<.01$, would be dismissed as error. These are all a priori considerations. The results we obtained, however, were so robust that it hardly mattered which type of correction one would prefer to apply.
To test whether the amplitudes of the SNs in the three discriminability conditions differed, two amplitude measures were obtained and tested. The SNs concerned those related to the selection of the easy-to-discriminate colors and shapes in the CESE, CHSE, and CESH conditions. First, a software algorithm determined the mean amplitude of the SN in the 200 - to $250-\mathrm{msec}$ poststimulus window. This window was determined on the basis of the grand-averaged ERP differences; it represents the initial $50 \mathrm{msec}$ of these differences (i.e., SNs). Second, the software algorithm determined the maximum amplitude of the $\mathrm{SN}$ in the 250- to 350 -msec poststimulus window. The mean and peak amplitudes found were then submitted to a MANOVA. Accuracy and response times (RTs) were also analyzed with a MANOVA, with discriminability as factor.

\section{RESULTS}

\section{Behavioral Measures}

The task was easy in all three discriminability conditions. When color and shape were easy to discriminate (the CESE condition), errors (misses and false alarms summed together) occurred on $0.60 \%$ of the trials. When the color was hard to discriminate (the CHSE condition), the error rate was $1.17 \%$; when the shape was hard to discriminate (the CESH condition), the error rate was $0.35 \%$. The number of errors was larger in the CHSE condition than in the CESE and CESH conditions $\left[F(1,17)=15.89, M S_{\mathrm{e}}=\right.$ $159, p<.001]$ and $\left[F(1,17)=13.08, M S_{\mathrm{e}}=173, p<.002\right.$, respectively], and it did not differ between the CESE and CESH conditions $[F(1,17)<1]$.

The averaged mean RTs in the CESE, CHSE, and CESH conditions were 417,460 , and $417 \mathrm{msec}$, respectively. As with the error rates, RT in CHSE was significantly longer than RT in CESE and CESH $[F(1,17)=$ $23.67, M S_{\mathrm{e}}=707, p<.0005$, and $F(1,17)=26.2, M S_{\mathrm{e}}=$ $656, p<.0005$, respectively], but it did not differ between CESE and CESH $\left[F(1,17)<1, M S_{\mathrm{e}}=666\right]$. These results show that the use of hard-to-discriminate color increased the latency and decreased the accuracy of the conjunction detection response, relative to the use of easyto-discriminate color (the CESE condition). They further show that the use of hard-to-discriminate shapes did not change the speed and accuracy of responses, relative to the use of easy-to-discriminate shapes (the CESE condition). Before discussing these results, we will first present the ERP findings.

\section{ERP Measures}

Color-Easy/Shape-Easy (CESE) condition. Figure 3 presents the grand-averaged ERPs elicited by the six stimulus types in the CESE condition at the central $\left(\mathrm{C}^{\prime}, \mathrm{Cz}\right.$, and $\mathrm{C}^{\prime}$ ) and posterior (TO1, $\mathrm{Pz}, \mathrm{TO} 2, \mathrm{O} 1, \mathrm{Oz}$, and $\mathrm{O} 2$ ) electrodes. Note that all stimuli elicited a positivenegative $\mathrm{P} 120-\mathrm{N} 190$ sequence at the occipital $(\mathrm{O} 1, \mathrm{Oz}$, and $\mathrm{O} 2$ ) and temporooccipital ( $\mathrm{TO} 1$ and $\mathrm{TO} 2$ ) electrodes. The amplitude of this positive-negative sequence was maximal at the TO1 and TO2 electrodes. Figure 3 also shows that the relevance of the various stimulus types started to produce differences in the ERPs just before $200 \mathrm{msec}$ (i.e., where the N190 peaks) at the (temporo-) occipital electrodes and after $200 \mathrm{msec}$ at the other elec- 


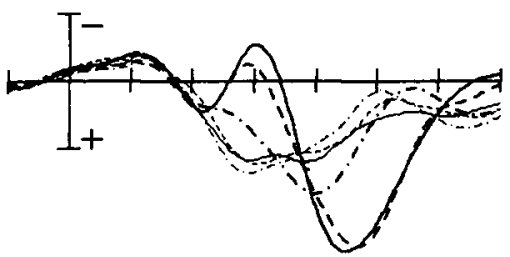

T01
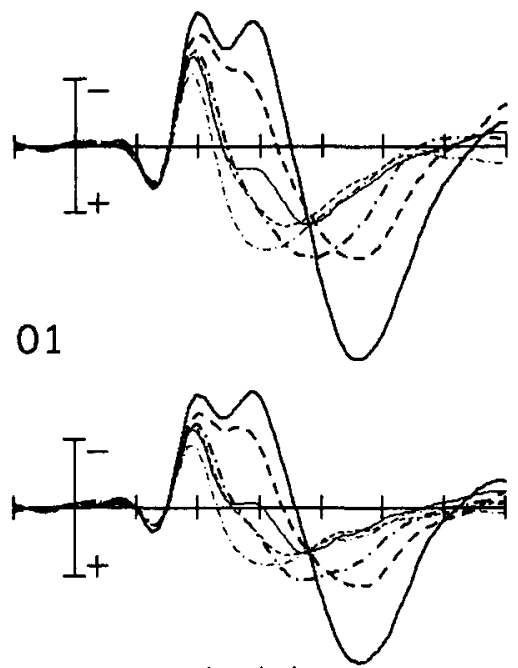

$-\mathbf{c}+\mathbf{s}+\mathbf{t}$

$---\mathbf{C}+\mathbf{S}+$

$\cdot \cdots \cdot \mathrm{C}+\mathrm{S}-$
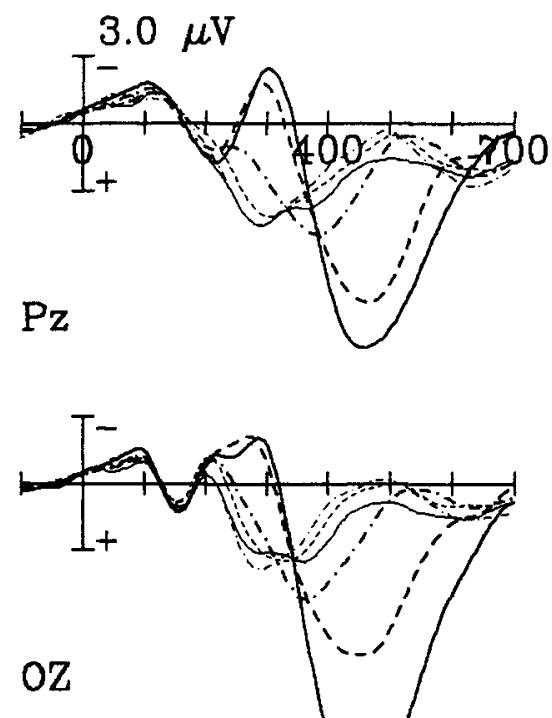

T02
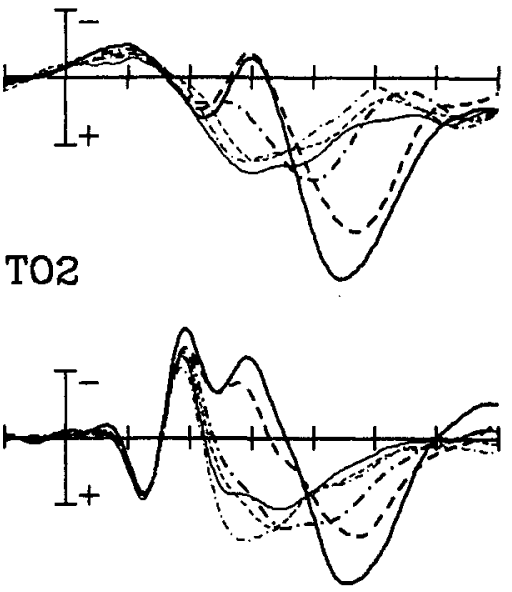

02

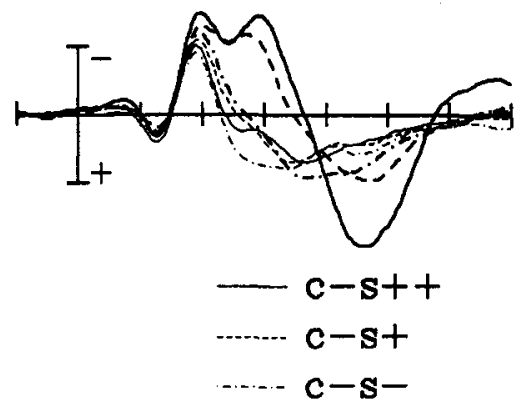

Figure 3. Grand-averaged ERP waveforms in microvolts obtained in the $\mathrm{CESE}$ condition at the central $\left(\mathrm{C3}^{\prime}, \mathrm{Cz}, \mathrm{C4}^{\prime}\right)$ and posterior (TO1, Pz, TO2, O1, Oz, O2) electrodes elicited by the $C+S++, C+S+, C+S-, C-S++, C-S+$, and $C-S-$ stimuli. Negative amplitudes are plotted upward.

trodes. These differences were maximal at the TO1 and TO2 electrodes and consisted of an increased and prolonged negativity to stimuli with one or more relevant attributes. As Figure 3 shows, the more attributes a stimulus shared with the target, the more the negativity starting in the N190 was prolonged. Because this negativity was earliest and largest at the occipital electrodes and was related to the relevance of the colors and the shapes, it fulfilled the criteria for identifying it in the ERPs as SN (e.g., Näätänen, 1992).

The differences between the ERPs - that is, the SNsare better illustrated in Figure 4. This figure shows the grand-average difference potentials collapsed over the left (TO1 electrode) and right (TO2 electrode) hemispheres. These were obtained by subtracting the ERPs elicited by two classes of stimuli that differed in the relevance of one stimulus attribute. Figure $4 \mathrm{~A}$ shows the difference between the ERPs that were elicited when the stimulus had a relevant versus an irrelevant color. This color relevance effect was present when the global shape of the stimulus was relevant $[\operatorname{ERP}(C+S+)-\operatorname{ERP}(C-S+)$; continuous waveform] and when the global shape was irrelevant $[\operatorname{ERP}(C+S-)-\operatorname{ERP}(C-S-)$; dashed waveform]. Figure 4B shows the difference between the ERPs that were produced when the stimulus had a relevant versus an irrelevant global shape. This global shape effect was present when the color was relevant $[\mathrm{ERP}(C+S+)-$ $\operatorname{ERP}(C+S-)$; continuous waveform] and when it was irrelevant $[\operatorname{ERP}(C-S+)-\operatorname{ERP}(C-S-)$; dashed waveform]. Figure $4 C$ presents the ERP differences due to the difference in relevance of the local shape of the stimulus. As with global shape, variation in local shape relevance also produced a difference in the ERPs both when the color was relevant $[\operatorname{ERP}(C+S++)-\operatorname{ERP}(C+S+)$; continuous waveform] and when it was irrelevant $[\operatorname{ERP}(C-S++)-$ ERP $(C-S+)$; dashed waveform]. Clearly, in all three panels, the amplitude of the attribute selection effects reached larger levels when the other attribute was relevant than when it was irrelevant.

Figure 5 shows the time ranges in which the hypothesis that 2 ERPs were identical was rejected on 3 or more consecutive 8 -msec epochs $(24 \mathrm{msec})$ for a number of comparisons. This figure shows that the $\mathrm{SN}$ related to color relevance started at about the same time $(196 \mathrm{msec})$ as 

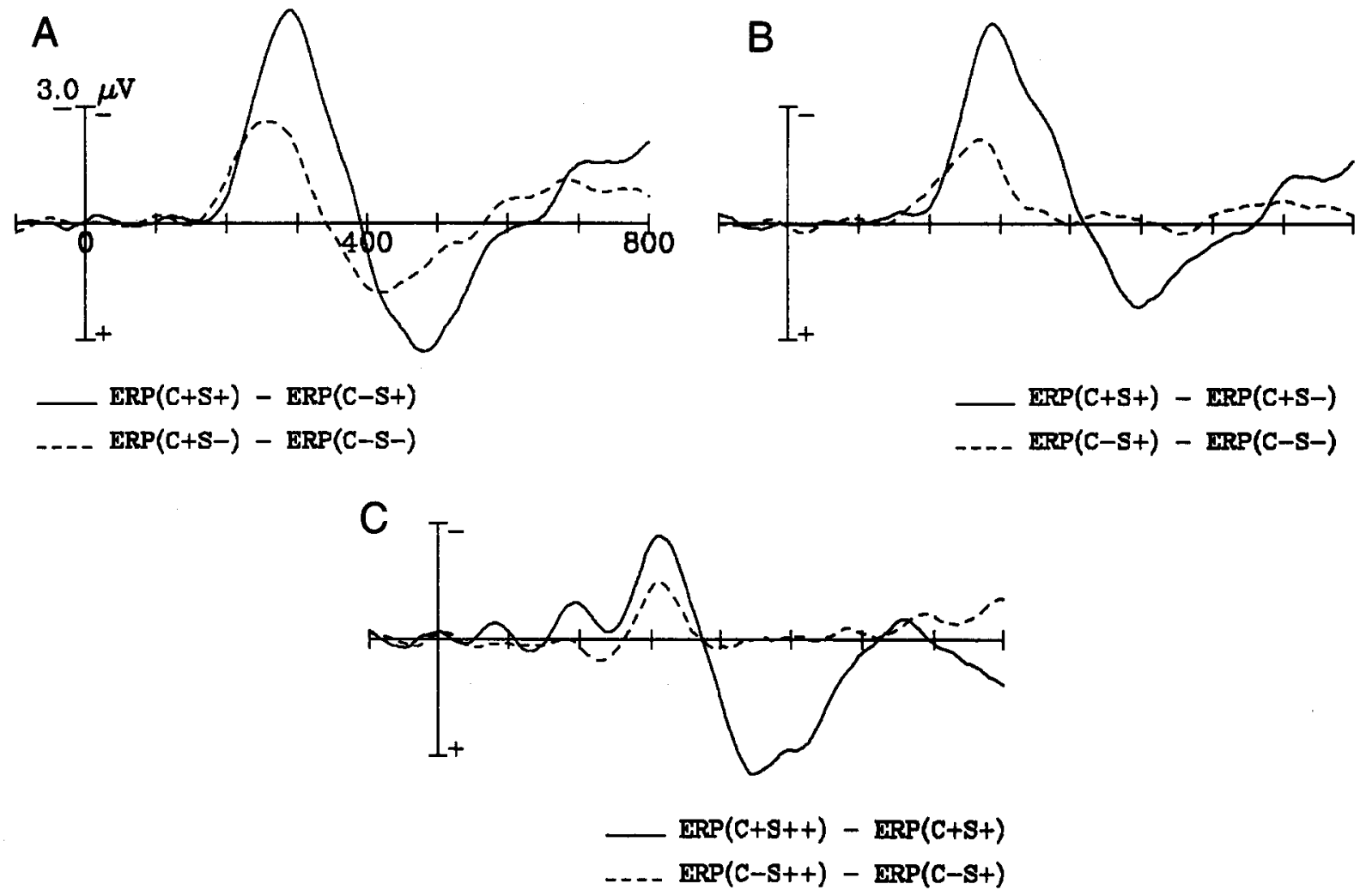

Figure 4. Grand-averaged difference potentials in microvolts obtained in the CESE condition. (A) The effects of color relevance when the global shape was relevant (continuous waveform) and was irrelevant (the dashed waveform). (B)The effects of global shape relevance when the color was relevant (continuous waveform) and was irrelevant (dashed waveform). (C) The effects of local shape relevance when the color was relevant (continuous waveform) and was irrelevant (dashed waveform). Negative amplitudes are plotted upward.

the SN related to global shape relevance ( $204 \mathrm{msec})$. At $252 \mathrm{msec}$, color relevance and global shape relevance started to interact, reflecting that the $\mathrm{SN}$ amplitudes due to color and shape relevance at that time became larger when the other attribute was relevant than when the other attribute was irrelevant (Figures $4 \mathrm{~A}$ and $4 \mathrm{~B}$ show this effect as the time where the continuous and dashed waveforms start to diverge). This suggests that processing became selective for the conjunction of color and global shape at about $252 \mathrm{msec}$. Note that, before SN onset, there was no interval in which 3 or more consecutive epochs yielded a significant difference for any of the comparisons.

A more fine-grained analysis tested the effect of relevance of an attribute at each level of the other attribute. The results of these tests are shown in the lower part of Figure 5. The effect of color relevance started at $212 \mathrm{msec}$ (duration $152 \mathrm{msec}$ ) when the global shape was relevant $[\operatorname{ERP}(C+S+)-\operatorname{ERP}(C-S+)]$ and at $188 \mathrm{msec}$ (duration $136 \mathrm{msec})$ when the global shape was irrelevant $[\operatorname{ERP}(C+S-)-\operatorname{ERP}(C-S-)]$. The effect of global shape relevance started at $220 \mathrm{msec}$ (duration $176 \mathrm{msec}$ ) when the color was relevant $[\mathrm{ERP}(C+S+)-\operatorname{ERP}(C+S-)]$ and at $220 \mathrm{msec}$ (duration $96 \mathrm{msec}$ ) when the color was irrelevant $[\operatorname{ERP}(C-S+)-\operatorname{ERP}(C-S-)]$. The effect of local shape relevance started at $276 \mathrm{msec}$ (duration $80 \mathrm{msec}$ ) when the color was relevant $[\operatorname{ERP}(C+S++)-\operatorname{ERP}(C+S+)]$ and at $300 \mathrm{msec}$ (duration $40 \mathrm{msec}$ ) when the color was irrelevant $[\operatorname{ERP}(C-S++)-\operatorname{ERP}(C-S+)]$.

Because the $C+S++$ stimuli were responded to and the $C+S+$ stimuli were not, one might want to propose that the $[\operatorname{ERP}(C+S++)-\operatorname{ERP}(C+S+)]$ difference (i.e., the local shape $\mathrm{SN}$ ) was due to effects on the ERP of motorrelated processing. This interpretation can be ruled out on several grounds. First, the $[\operatorname{ERP}(C+S++)-\operatorname{ERP}(C+S+)]$ difference was parallelled by an $[\operatorname{ERP}(C-S++)-$ $\operatorname{ERP}(C-S+)]$ difference with equal scalp distribution and timing. So, even when no responses were made (to $C-S++$ and $C-S+$ stimuli), the same effect was obtained. Second, at the $\mathrm{C}^{3}$ ' electrode placed over the motor cortex related to right-hand responding, the difference between $C+S++$ and $C+S+$ stimuli was about one third as large as, and started later than, that at the TO1 electrode. We know from previous research that motor-related effects in the ERP on right-hand response trials are maximal at $\mathrm{C}^{\prime}$ and are much smaller or absent at more posterior electrodes (e.g., Kutas \& Donchin, 1980).

In summary, the present findings suggest that color and global shape were identified and selected at about the same time (196-204 msec), that local shape was identified and selected later $(276 \mathrm{msec})$, and that pro- 

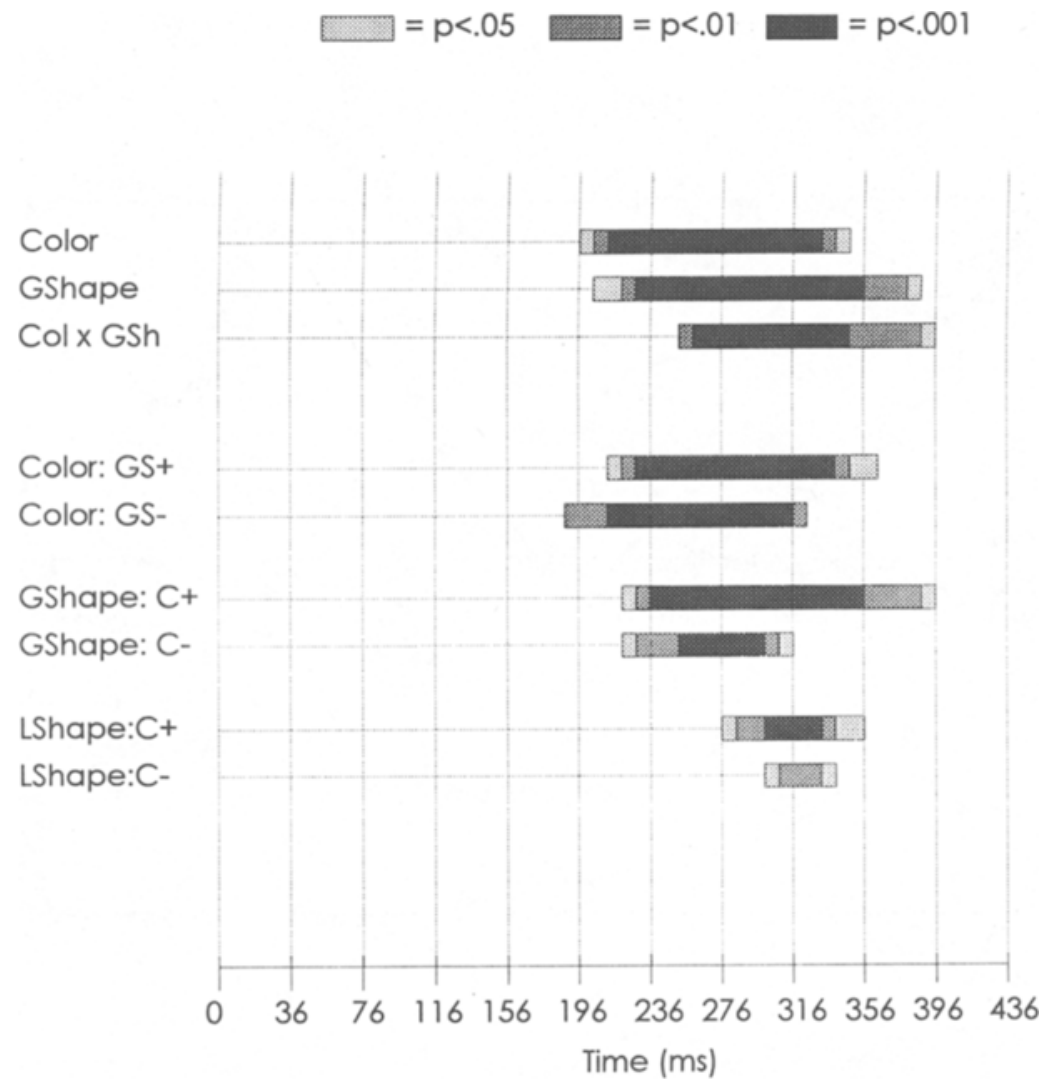

\begin{abstract}
Figure 5. Effects of attention on the $\mathrm{SN}$ in the CESE condition (see introduction of this paper) in the initial $436 \mathrm{msec}$ after stimulus presentation. Epochs of $8 \mathrm{msec}$ in which the SN had a significant amplitude are depicted with different hatching of texture depending on the level of significance (see legend). The rows labeled Color, GShape, and Col $\times$ GSh show the main effects of color, global shape, and their interaction, respectively. The rows labeled Color: GS+ and Color: GS - show the subeffects of color relevance when the global shape was relevant and when it was irrelevant, respectively. The rows labeled GShape: $C+$ and GShape: $C$ - show the subeffects of global shape relevance when the color was relevant and when it was irrelevant, respectively. The rows labeled LShape: $C+$ and LShape: $C$ - show the subeffects of local shape relevance when the color was relevant and when it was irrelevant, respectively.
\end{abstract}

cessing became specific for the $C+S+$ conjunction at $252 \mathrm{msec}$. The findings further confirm the predictions derived from the exhaustive model, and they are inconsistent with the predictions derived from both the selfterminating model and the holistic model. This can easily be verified by comparing the present findings with the predictions listed in Table 1 (substitute $C$ for $A$ and substitute $S$ for $B$ to obtain the relevant ERP differences). Both the self-terminating model in which color and global shape are identified equally fast and the holistic model predict that $(3)[\operatorname{ERP}(C+S-)-\operatorname{ERP}(C-S-)]=$ 0 and (4) $[\operatorname{ERP}(C-S+)-\operatorname{ERP}(C-S-)]=0$, in the time interval in which an SN can occur $(120-320 \mathrm{msec}$, see above). The self-terminating model in which color is identified earlier than is global shape predicts that (4) $[\operatorname{ERP}(C-S+)-\operatorname{ERP}(C-S-)]=0$ (i.e., no global shape relevance effect when the color is irrelevant). We found, however, that all four difference equations (Predictions 1-4) became larger than zero in the SN time in- terval, which is predicted only by the exhaustive model. We further found that (1) initially, the relevance effects of color and global shape were additive, confirming Predictions 5 and 6 of the exhaustive model, and (2) the color and global shape relevance effects started to interact at $252 \mathrm{msec}$, confirming Predictions 7 and 8 of the exhaustive model. In addition, we found that $[\operatorname{ERP}(C-S++)-$ $\operatorname{ERP}(C-S+)]>0$ (i.e., an effect of local shape relevance when the color was irrelevant), which is a special case of Prediction 4, $[\operatorname{ERP}(C-S+)-\operatorname{ERP}(C-S-)]>0$. We discuss these findings in terms of two-stage models of multiattribute selection after presenting the results in the CHSE and CESH conditions.

Color-Hard/Shape-Easy (CHSE) condition. Figure 6 presents the grand-averaged ERPs obtained in the CHSE condition at the central and posterior electrodes. As in the CESE condition, all stimuli produced a positive-negative sequence that was maximal at the TO1 and TO2 electrodes. The ERPs started to differ as a function 
C3'

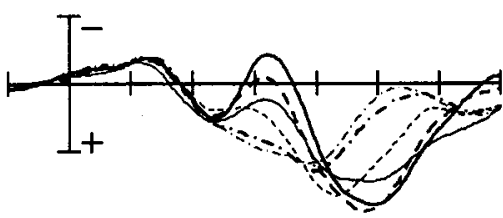

T01

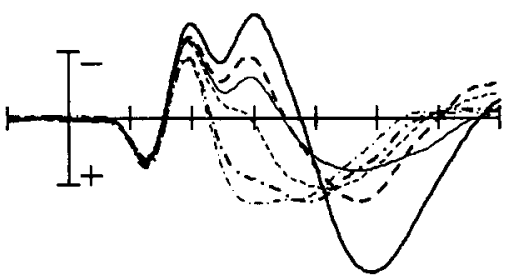

01

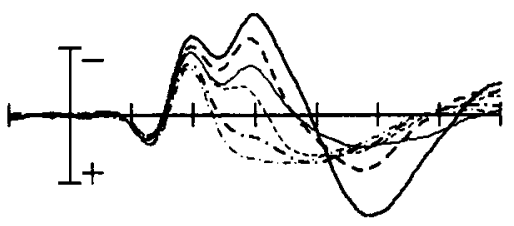

$$
\begin{array}{ll}
-c+s++ \\
\cdots & c+s+ \\
\cdots & c+s-
\end{array}
$$

$\mathrm{Cz}$

$\mathrm{C} 4^{\prime}$

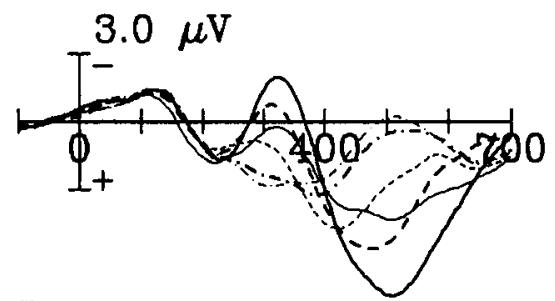

$\mathrm{Pz}$

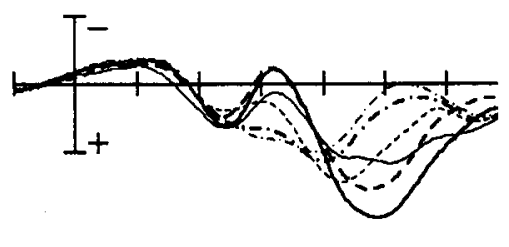

TO2
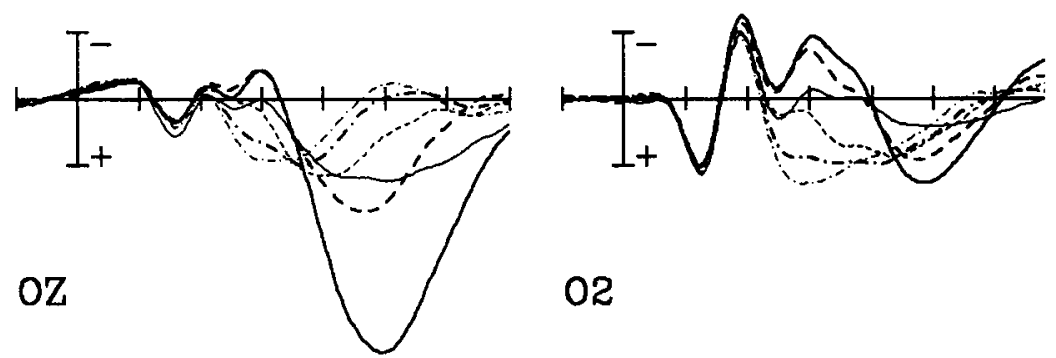

02
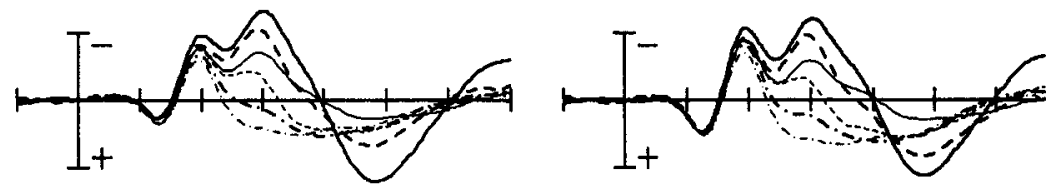

Figure 6. Grand-averaged ERP waveforms in microvolts obtained in the CHSE condition. See Figure 3 caption for explanation of abbreviations.

of stimulus relevance in the N190 peak; as Figure 6 clearly shows these differences were again largest and earliest at the TO electrodes.

Figure 7 shows the difference potentials collapsed over hemispheres (i.e., over TO1 and TO2). Figure 7A shows effects of color relevance both when the global shape was relevant and when it was irrelevant. Figures $7 \mathrm{~B}$ and $7 \mathrm{C}$ show that large effects of shape selection were present both when the color was relevant and when it was irrelevant.

Figure 8 shows the time ranges in which these effects were significant. This figure shows that the $\mathrm{SN}$ related to color relevance started at $252 \mathrm{msec}$. The effect of global shape relevance started at $164 \mathrm{msec}$. At $284 \mathrm{msec}$, these effects started to interact, reflecting that the SN amplitudes due to color and shape relevance at that time became larger when the other attribute was relevant than when it was irrelevant (see also Figure 7). This suggests that processing became selective for the conjunction of color and global shape at $284 \mathrm{msec}$.

The tests of the effect of relevance of an attribute at each level of the other attribute revealed the following significant $\mathrm{SN}$ intervals. The effect of color relevance started at $268 \mathrm{msec}$ (duration $152 \mathrm{msec}$ ) when the shape was rel- evant and at $244 \mathrm{msec}$ (duration $96 \mathrm{msec}$ ) when the shape was irrelevant. The effect of global shape relevance started at $196 \mathrm{msec}$ (duration $224 \mathrm{msec}$ ) when the color was relevant and at $164 \mathrm{msec}$ (duration $176 \mathrm{msec}$ ) when the color was irrelevant. The effect of local shape relevance started at $300 \mathrm{msec}$ (duration $72 \mathrm{msec}$ ) when the color was relevant and at $292 \mathrm{msec}$ (duration $176 \mathrm{msec}$ ) when the color was irrelevant. The early effects of global shape (onset $164 \mathrm{msec}$ ) were small and short (see Figure 7). They may have been caused by a few trials or a few subjects having deviantly large amplitudes at that time ("outlier" effect). The large global shape SNs in Figure 7 produce statistically robust effects starting at $204 \mathrm{msec}$ (see Figure 8, GShape effect) and at $212 \mathrm{msec}$ (see Figure 8, GShape: $C$ - effect).

These findings suggest that color was identified and selected later than was global shape (252 and $204 \mathrm{msec}$, respectively), that local shape was identified and selected last ( $292 \mathrm{msec})$, and that conjunction-specific processing started at $284 \mathrm{msec}$. As in the CESE condition, the findings in this condition confirm the predictions derived from the exhaustive model (see Table 1, substitute $S$ for $A$ and substitute $C$ for $B$, because global shape was 
A

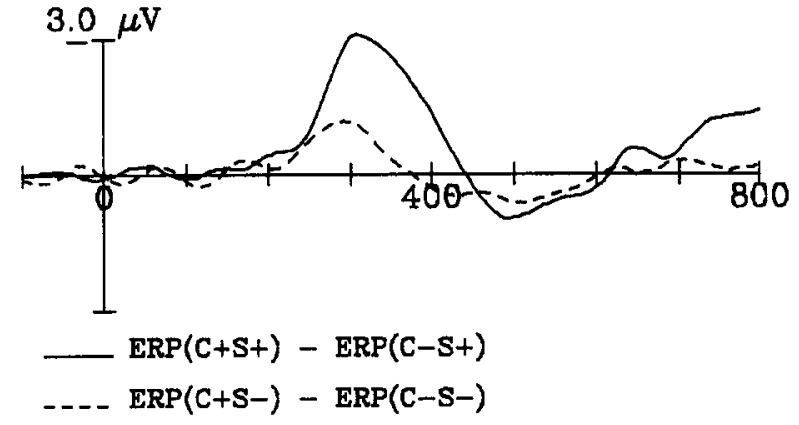

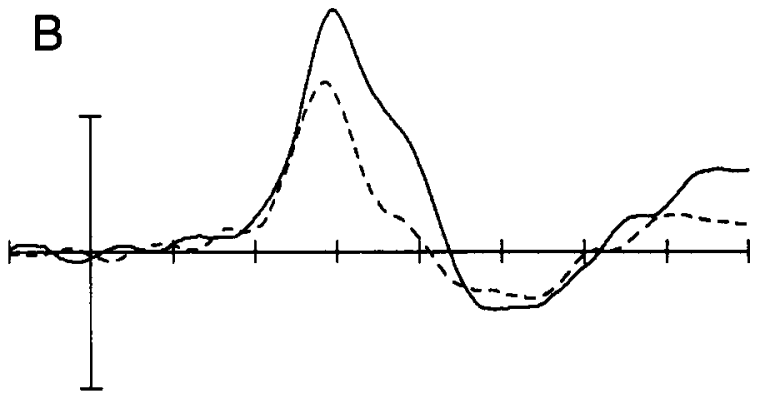

$-\operatorname{ERP}(\mathrm{C}+\mathrm{S}+)-\operatorname{ERP}(\mathrm{C}+\mathrm{S}-)$

-.. $\operatorname{ERP}(\mathrm{C}-\mathrm{S}+)-\operatorname{ERP}(\mathrm{C}-\mathrm{S}-)$

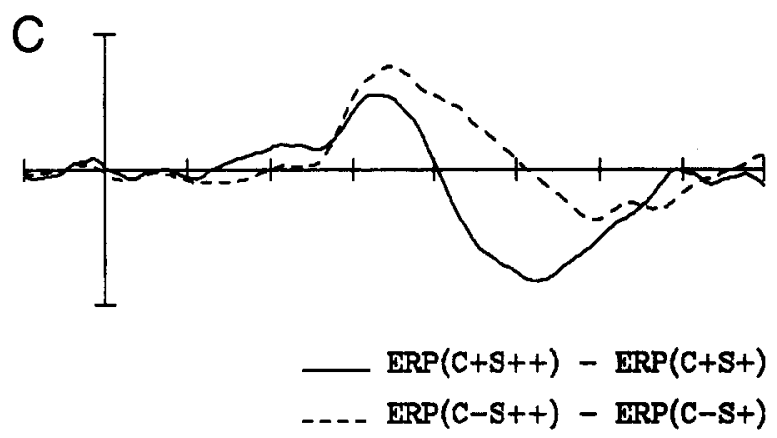

Figure 7. Grand-averaged difference potentials in microvolts obtained in the CHSE condition. See Figure 4 caption for further explanation.

identified earlier than was color). All difference equations (Predictions 1-4) became larger than zero in the SN time interval. The findings violate Prediction 4 derived from a self-terminating model in which global shape is identified earlier than is color. This means that there was a color relevance effect when the global shape was irrelevant, despite the fact that the global shape was identified much earlier and thus could have been easily used to terminate any further processing. The findings further violate Predictions 3 and 4 derived from the holistic model.

Color-Easy/Shape-Hard (CESH) condition. Figure 9 presents the grand-averaged ERPs obtained in the CESH condition. The P120-N190 sequence is again largest at the TO electrodes, and the differences between the ERPs as a function of stimulus relevance are again largest and earliest at these electrodes. Note the relatively large effects of local shape relevance for stimuli with relevant colors $(C+S++$ vs. $C+S+)$ and for those with irrelevant colors $(C-S++$ vs. $C-S+)$.

Figure 10 shows the difference potentials collapsed over the TO1 and TO2 electrodes. Figure 10A shows that large SNs related to color selection were present when the shape was relevant (continuous waveform) and when the shape was irrelevant (dashed waveform). Figure 10B shows that only an $\mathrm{SN}$ related to global shape selection was present when the color was relevant. Figure 10C shows that large SNs related to local shape selection were pres- ent both when the color was relevant and when the color was irrelevant.

Figure 11 shows the time ranges in which these effects were significant. This figure shows that the $\mathrm{SN}$ related to color relevance started at $196 \mathrm{msec}$, and the SN related to global shape relevance started at $260 \mathrm{msec}$. At $268 \mathrm{msec}$, these effects started to interact, reflecting that the SN amplitudes due to color and shape relevance at that time became larger when the other attribute was relevant (see also Figure 10). This suggests that processing became selective for the conjunction of color and shape at about $268 \mathrm{msec}$. As Figure 11 shows, the color $\times$ global shape interaction (Col $\times$ GSh) that started at $268 \mathrm{msec}$ was interrupted after 3 epochs and later became significant again. Figure 10A shows why. At about $250 \mathrm{msec}$, the color relevance SNs for the relevant (continuous waveform) and irrelevant (dashed waveform) global shapes started to diverge, but converged again at about $290 \mathrm{msec}$, after which they again diverged. A similar effect can be seen in Figure 10C (see also Figures 4 and 7).

The tests of the effect of relevance of an attribute at each level of the other attribute revealed the following significant SN intervals. The effect of color relevance started at $204 \mathrm{msec}$ (duration $160 \mathrm{msec}$ ) when the shape was relevant and at $204 \mathrm{msec}$ (duration $136 \mathrm{msec}$ ) when the shape was irrelevant. The effect of global shape relevance started at $260 \mathrm{msec}$ (duration $152 \mathrm{msec}$ ) when the color was relevant; there was no significant effect of 

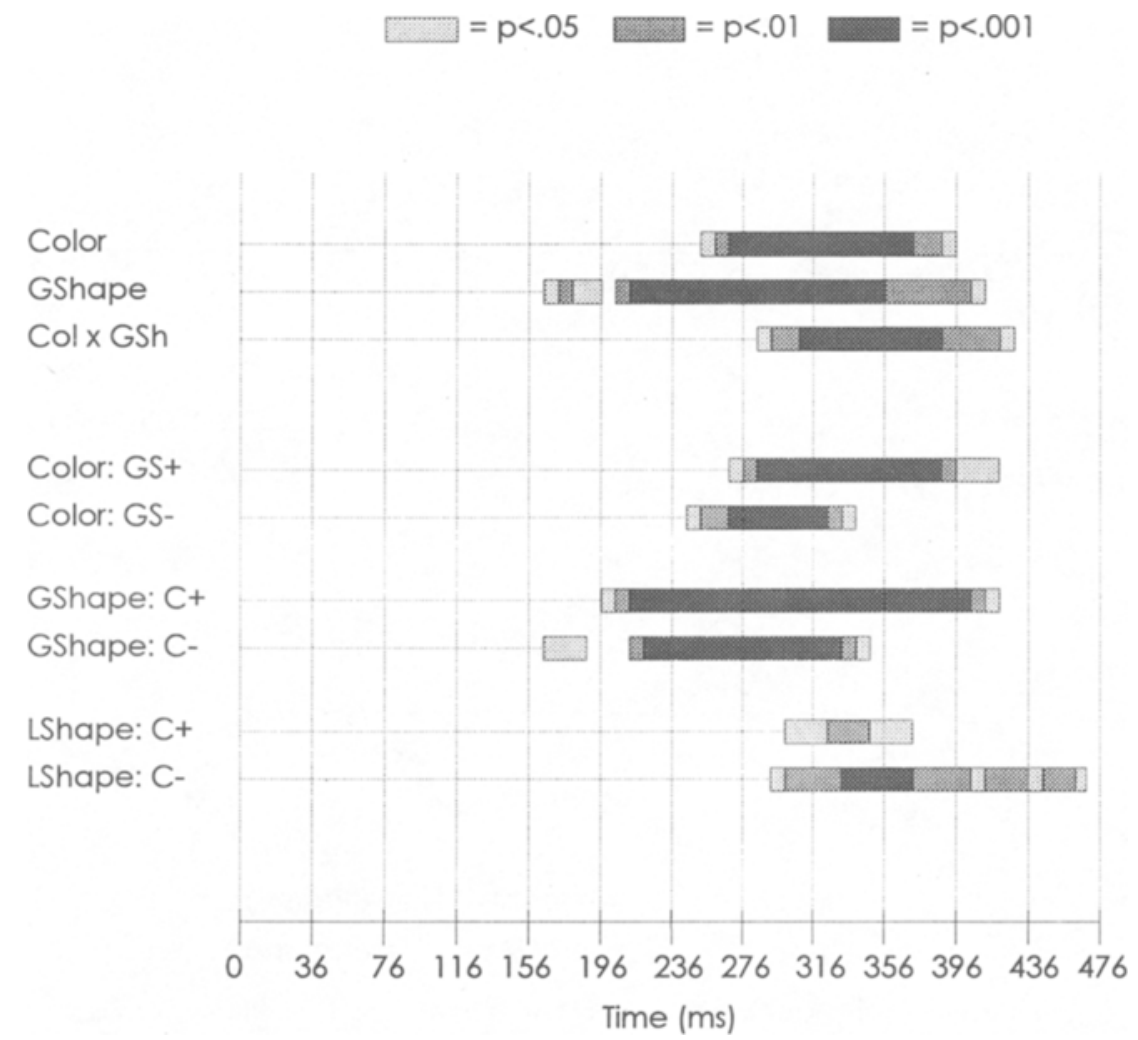

Figure 8. Effects of attention on the $\mathrm{SN}$ in the CHSE condition (see introduction of this paper) in the initial 476 msec after stimulus presentation. See Figure 5 caption for further explanation.

global shape when the color was irrelevant. The effect of local shape relevance started at $268 \mathrm{msec}$ (duration $96 \mathrm{msec}$ ) when the color was relevant; there was a small initial significant interval that started at $188 \mathrm{msec}$ (duration $32 \mathrm{msec}$ ) and a later interval that started at $260 \mathrm{msec}$ (duration $136 \mathrm{msec}$ ) when the color was irrelevant. As Figure 10 shows, the small initial effect concerned a small dip in the difference potential preceding the large $\mathrm{SN}$ we are interested in; therefore, we take $260 \mathrm{msec}$ (the onset of the second interval) as the onset of the SN related to local shape.

These findings suggest that color was identified and selected earlier than was global shape $(196$ and $260 \mathrm{msec}$, respectively), that global and local shape were identified and selected at about the same time $(260 \mathrm{msec})$, and that conjunction-specific processing started at $268 \mathrm{msec}$. In this condition, there was a global shape $\mathrm{SN}$ when the color was relevant but not when the color was irrelevant. There was a local shape SN both when the color was relevant and when it was not. These results support selfterminating processing of global shape and exhaustive processing of local shape (see Table 1, substitute $C$ for $A$ and substitute $S$ for $B$, because color was identified earlier than were global and local shapes). That is, the $[\operatorname{ERP}(C-S+)-\operatorname{ERP}(C-S-)]$ difference was zero in the $\mathrm{SN}$ interval, confirming Prediction 4 of the selfterminating model, but the $[\operatorname{ERP}(C-S++)-\operatorname{ERP}(C-S+)]$ difference was significantly larger than zero, which confirms Prediction 4 of the exhaustive model.

\section{Amplitude Measures of the SN}

Figure 12 shows a comparison of the SNs related to discriminating parallel identification and a mixture of trials on which color and shape are identified in series. The mixture model of serial trials predicts smaller easy-todiscriminate-attribute SNs of longer duration in the CESE condition than in the other conditions. The parallel model predicts no difference. Figure 12A shows the difference waves related to color selection in the two color-easy (CESE and CESH) conditions. These concern the $[\mathrm{ERP}(C+S+)-\operatorname{ERP}(C-S+)]$ differences. Figure 12B shows the difference waves related to global shape selection in the two shape-easy (CESE and CHSE) conditions. These concern the $[\operatorname{ERP}(C+S+)-\operatorname{ERP}(C+S-)]$ differences. As Figure 12 shows, the differences in the CESE condition were not smaller nor had they a longer duration than did those in the CESH and CHSE conditions.

To test whether the initial $50 \mathrm{msec}$ of the SNs and their peak amplitudes differed between the CESE on the one hand and CHSE and CESH conditions on the other, we used the mean and peak amplitudes of the ERP differences involving the easy-to-discriminate stimulus attributes when the complementary attribute was relevant. The reason for this selection was that these differences 


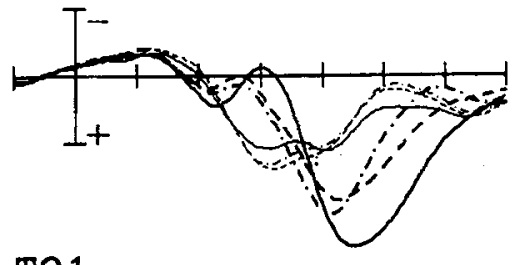

T01
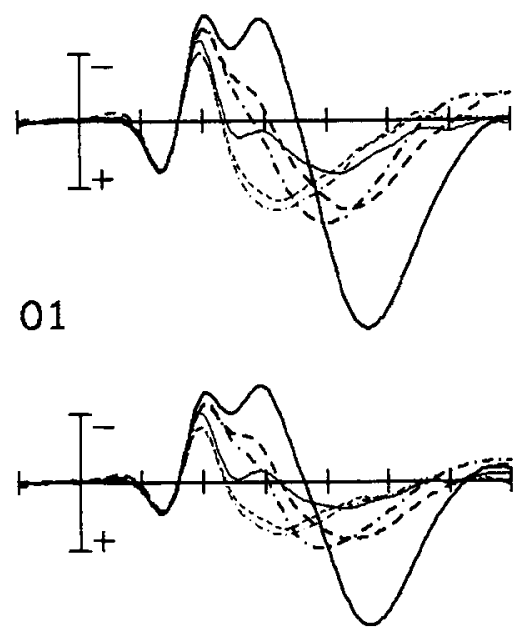

$$
\begin{aligned}
& -c+s++ \\
& \ldots c+s+ \\
& \ldots \ldots c+s-
\end{aligned}
$$

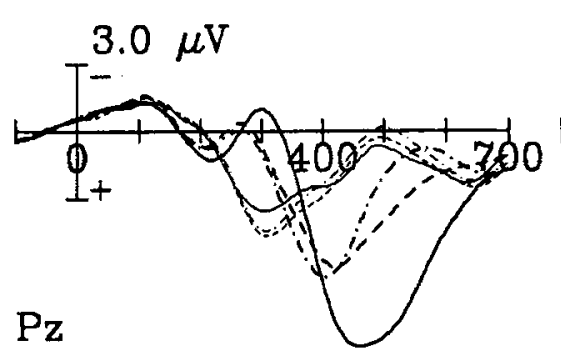

TO2
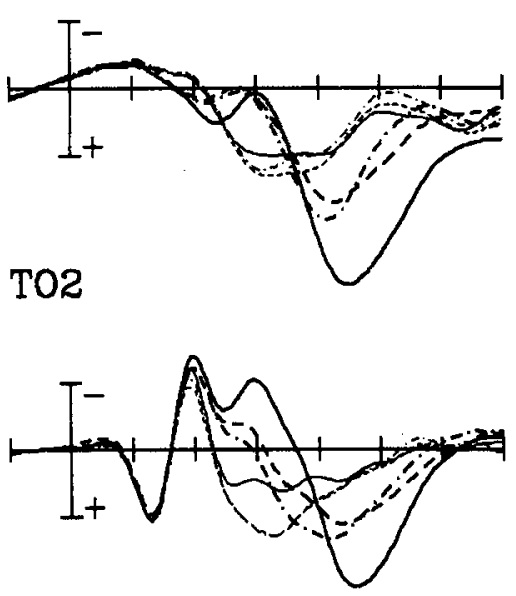

02
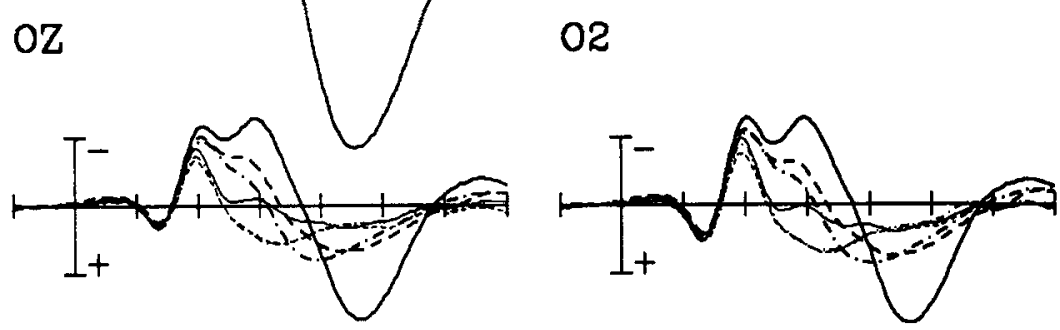

Figure 9. Grand-averaged ERP waveforms in microvolts obtained in the CESH condition. See Figure 3 caption for the explanation of the abbreviations.

would be affected the least by the discriminability factor. In the 50-msec window after their onset, the grandaveraged $[\operatorname{ERP}(C+S+)-\operatorname{ERP}(C-S+)]$ difference amplitudes in the CESE and CESH conditions were 2.60 and $2.20 \mu \mathrm{V}$, respectively. This difference was not significant $\left[F(1,17)<1, M S_{\mathrm{e}}=1.92\right]$. In this window, the grand-averaged $[\mathrm{ERP}(C+S+)-\operatorname{ERP}(C+S-)]$ difference amplitudes in the CESE and CHSE conditions were 1.80 and $1.71 \mu \mathrm{V}$, respectively. This difference also was not significant $\left[F(1,17)<1, M S_{\mathrm{e}}=0.89\right]$. The grand-averaged $[\operatorname{ERP}(C+S+)-\operatorname{ERP}(C-S+)]$ peak amplitudes in the CESE and CESH conditions were 7.04 and $5.24 \mu \mathrm{V}$, respectively. This difference was significant $[F(1,17)=$ $18.62, M S_{\mathrm{e}}=1.55, p<.0005$ ], indicating that the colorrelated SN was smaller in the CESH condition. The grandaveraged $[\operatorname{ERP}(C+S+)-\operatorname{ERP}(C+S-)]$ peak amplitudes in the CESE and CHSE conditions were 6.73 and $6.64 \mu \mathrm{V}$, respectively. This difference was not significant $[F(1,17)<$ $\left.1, M S_{\mathrm{e}}=1.20\right]$. These results indicate that the initial 50 -msec mean amplitudes and peak amplitudes of the $[\operatorname{ERP}(C+S+)-\operatorname{ERP}(C-S+)]$ and $[\operatorname{ERP}(C+S+)-$
$\operatorname{ERP}(C+S-)]$ differences in the CESE condition were not smaller than the $[\operatorname{ERP}(C+S+)-\operatorname{ERP}(C-S+)]$ difference in the CESH condition and the $[\operatorname{ERP}(C+S+)-$ $\operatorname{ERP}(C+S-)]$ difference in the CHSE condition. These results indicate that the averaged color and global shape $\mathrm{SNs}$ in the CESE condition were not smaller than the color SN in CESH and the global shape SN in CHSE. This suggests, as will be discussed below, that a trial-mixture model of serial color and shape identification is highly unlikely.

\section{DISCUSSION}

In this study, we investigated three questions concerning multidimensional selection when the color and the shape of stimuli serve as selection cues: (1) whether the identification and selection of color and shape occur in parallel or in series, (2) whether the selection of color and shape occurs in a self-terminating and dependent manner or in an exhaustive and independent manner, and (3) whether the stage of feature integration can start only after the stage of separate attribute analyses has finished. 
A

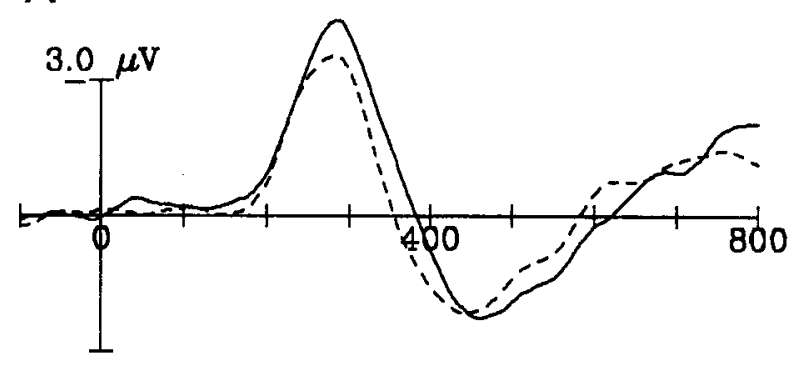

$\operatorname{ERP}(\mathrm{C}+\mathrm{S}+)-\operatorname{ERP}(\mathrm{C}-\mathrm{S}+)$
$---\operatorname{ERP}(\mathrm{C}+\mathrm{S}-)-\operatorname{ERP}(\mathrm{C}-\mathrm{S}-)$
B

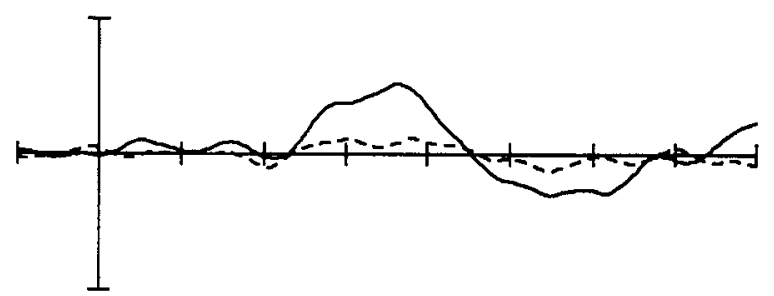

$\operatorname{ERP}(\mathrm{C}+\mathrm{S}+)-\operatorname{BRP}(\mathrm{C}+\mathrm{S}-)$

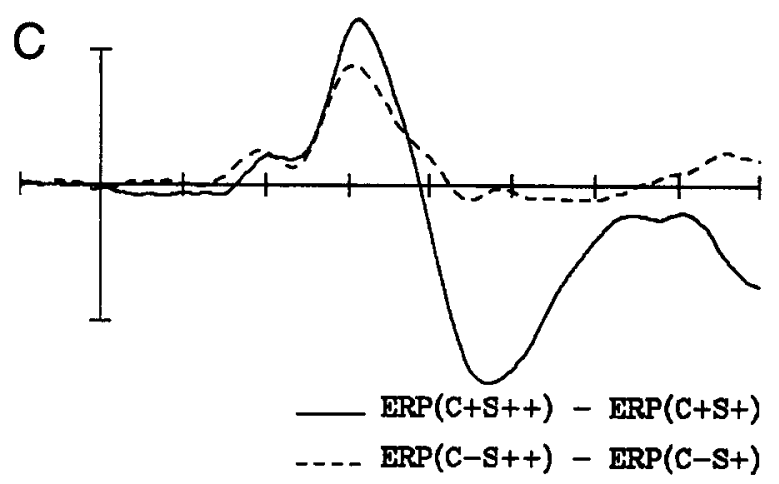

Figure 10. Grand-averaged difference potentials in microvolts obtained in the CESH condition. See Figure 4 caption for further explanation.

Figure 13 summarizes the main results we obtained. In this discussion, the onset latencies of the $\mathrm{SN}$ are rounded to the nearest $10 \mathrm{msec}$.

We derived SNs from the ERPs to conjunctions of color, global shape, and local shape. These conjunctions varied in the combination of attribute values they shared with a target conjunction. RTs were obtained for the conjunction targets. In different conditions, the relevant and irrelevant values of the color and the shape were all easy to discriminate (CESE), the color was hard to discriminate and the shape was easy to discriminate (CHSE), or the shape was hard to discriminate and the color easy to discriminate (CESH). In all three conditions, we obtained highly significant SNs related to color, global shape, and local shape selection. When we decreased the discriminability of the color, the onset latency of the SN related to color selection increased $50 \mathrm{msec}$. Decreasing the discriminability of (global) shape increased the onset latency of the SN related to (global) shape selection $60 \mathrm{msec}$. These increases in SN onset latencies closely parallelled the RT increases we obtained in the preliminary disjunctive RT experiment in which participants discriminated either color alone or global shape alone. In that experiment, the RT differences obtained for easyand hard-to-discriminate colors and for easy- and hardto-discriminate global shapes were 48 and $52 \mathrm{msec}$, respectively (see note 3 ). The discriminability manipulation thus resulted in highly consistent effects on the single-dimensional discrimination RTs and the SN onset latencies related to the single-dimensional analyses in the multidimensional task. The conjunctive RTs obtained in the multidimensional task were different. Decreasing color discriminability did increase conjunctive RT, but decreasing (global) shape discriminability did not increase conjunctive RT, both relative to the CESE condition. The reason for this dissociation is not clear, but it seems related to stages of processing that occur later than those tapped with the SN measure.

The pattern of the early ERP results supports the inference that color, global shape, and local shape were identified and selected in parallel, at least when they were relatively easy to discriminate. First, when both attributes were easily discriminable (CESE), we found that the onset latencies of the SNs related to color and global shape selection were about equal ( $200 \mathrm{msec})$. This supports a parallel model in which the identification of color and that of global shape have equal completion times, and in which both attributes are selected in parallel for further, task-directed processing. The equal onset latencies are inconsistent with a serial model in which identification of color and that of global shape were performed one after the other. 

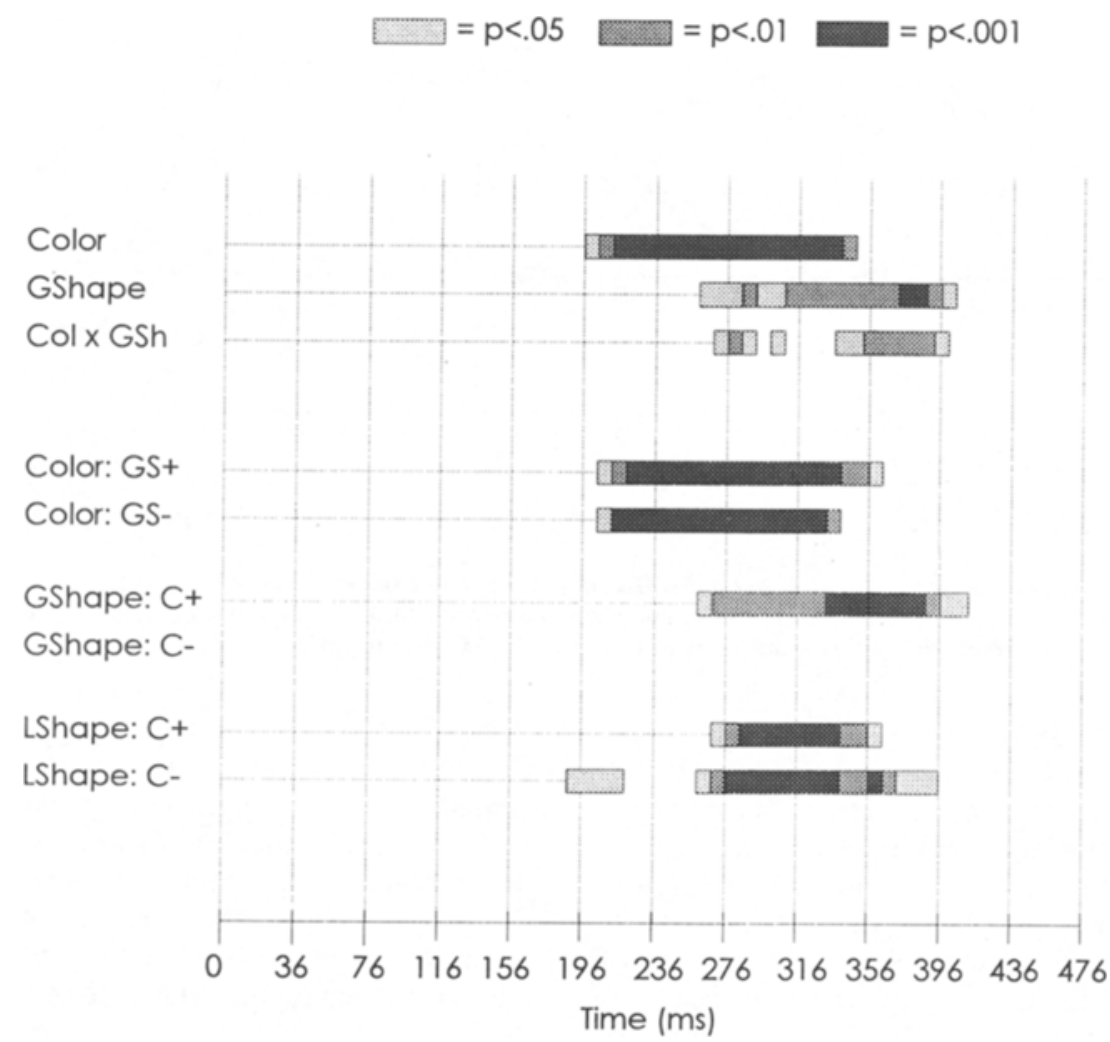

Figure 11. Effects of attention on the SN in the CESH condition (see introduction of this paper) in the initial $\mathbf{4 7 6}$ msec after stimulus presentation. See Figure 5 caption for further explanation.

Second, in the CESE condition, the SN related to local shape selection started later $(290 \mathrm{msec})$ than did the SNs related to color and global shape selection $(200 \mathrm{msec})$. Relative to the CESE condition, decreasing the discriminability of the color (CHSE) and the global shape (CESH) substantially increased the onset latency of the colorrelated SN (from 200 to $250 \mathrm{msec}$ ) and the global-shaperelated SN (from 200 to $260 \mathrm{msec}$ ), but not the onset latency of the SN related to local shape selection (from $290 \mathrm{msec}$ in CESE to $300 \mathrm{msec}$ in CHSE and $260 \mathrm{msec}$ in CESH). If local shape identification would have been performed in series after the analyses of color and global shape had finished, we should have found an increase in local shape $\mathrm{SN}$ onset latency, comparable to the increase in the color and global shape SN onset latencies, when color and shape were made harder to discriminate (see Figures $1 \mathrm{~A}$ and $1 \mathrm{~B}$ ). This was not the case, however. The identification of local shape seems therefore to have occurred in parallel with color and global shape analysis.

Third, the evidence contradicts an alternative explanation for the equal onsets of the color and global shape SNs in the CESE condition, which is based on a trialmixture model of serial color and shape identifications. As argued in the introduction, the occurrence of equal $\mathrm{SN}$ onset latencies related to multiple-attribute selections can, without additional evidence, be accounted for by a serial model. This model proposes that on about half of the trials, color is selected before global shape and that, on the other half of the trials, global shape is selected before color. After averaging across these trials, the SNs related to color and global shape selection would appear to start at the same time.

Another prediction derived from this model is, however, that the color-related SNs would be elicited at a substantially earlier time point on color-first trials than on global-shape-first (i.e., color-second) trials. Averaging the color SNs across these two trial types would have smeared out their amplitudes over a relatively long time range, resulting in a color $\mathrm{SN}$ of much smaller amplitude and of longer duration than the color SNs from each trial type separately (and the same is true for the global-shaperelated SNs on color-first and global-shape-first trials). Thus, if we could separate color-first trials and globalshape-first trials, we could test this alternative. This separation was accomplished with the discriminability manipulation. Because of the difference in discriminability between color and shape in the CHSE and CESH conditions, we obtained global-shape-first trials in the CHSE condition and color-first trials in the CESH condition. We used the color-related SN in the CESH and the global- 

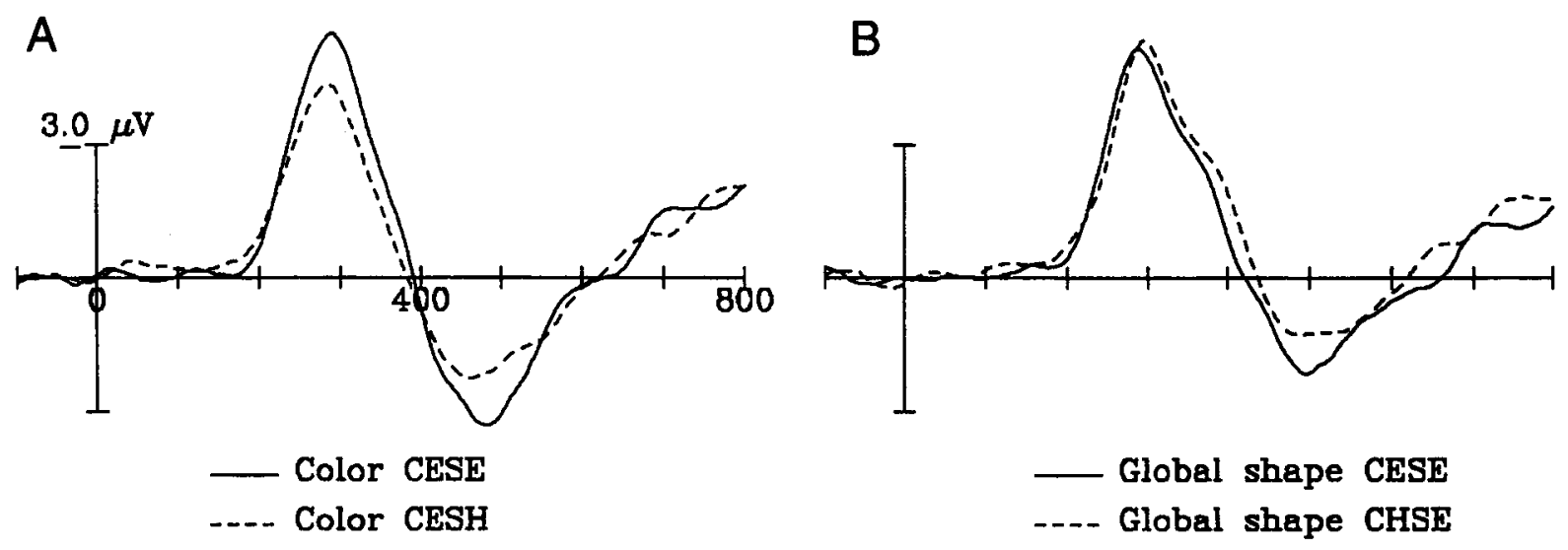

Figure 12. Grand-averaged difference potentials in microvolts concerning color relevance $[\mathbf{E R P}(C+S+)-\mathbf{E R P}(C-S+)]$ and global shape relevance $[\operatorname{ERP}(C+S+)-\operatorname{ERP}(C+S-)]$. (A) The color relevance effects obtained in the two color-easy (CESE and $C E S H)$ conditions. (B) The global shape effects in the two shape-easy (CESE and CHSE) conditions.

shape-related SN in the CHSE conditions as estimates of the color SN on color-first trials and the global shape SN on global-shape-first trials, respectively.

We found that the durations of the $[\operatorname{ERP}(C+S+)-$ $\operatorname{ERP}(C-S+)]$ differences in the CESE and CESH conditions were 150 and $160 \mathrm{msec}$, respectively, and those of the $[\operatorname{ERP}(C+S+)-\operatorname{ERP}(C+S-)]$ differences in the CESE and CHSE conditions were 180 and $220 \mathrm{msec}$, respectively. In addition we found that the initial $50-\mathrm{msec}$ mean amplitudes and the peak amplitudes of the SNs related to color and global shape selection in the CESE condition were not smaller than the SN amplitude related to color selection in the CESH condition and the SN amplitude related to global shape selection in the CHSE condition. These findings are fully inconsistent with the alternative trial-mixture serial model.

We infer, therefore, from these findings that, if relatively easy to discriminate, then color, global shape, and local shape, all tied to a single object, are identified and selected in parallel, and that a serial model cannot explain the present results. Can this inference be generalized to the analyses of color and shape when they differed in discriminability? The evidence shows that the SN onset latencies related to local shape selection in the CHSE and CESH conditions were not longer than those in the CESE condition, whereas the color and global shape SN onset latencies clearly increased. This supports the inference that, in all three conditions, local shape was identified in parallel with color and global shape. However, we cannot exclude that color and global shape were identified in a serial manner in the CHSE and CESH conditions. Making one of these attributes harder to discriminate may have induced the participants to adopt another processing strategy, in which the easy-to-discriminate attribute is identified first. Felfoldy and Garner (1971; see also Garner, 1974) reported evidence consistent with changes in strategy as a result of manipulating discriminability.

A second issue addressed in this study was whether the selection of color and shape occurs in a self-terminating and dependent manner or in an exhaustive and independent manner. The evidence bearing on this issue concerns the effects of relevance of color and shape as a function of the relevance of the complementary attribute. More specifically, the question to be answered was whether or not the amplitude of the SN related to the selection of an attribute depended on the relevance of the other attribute.

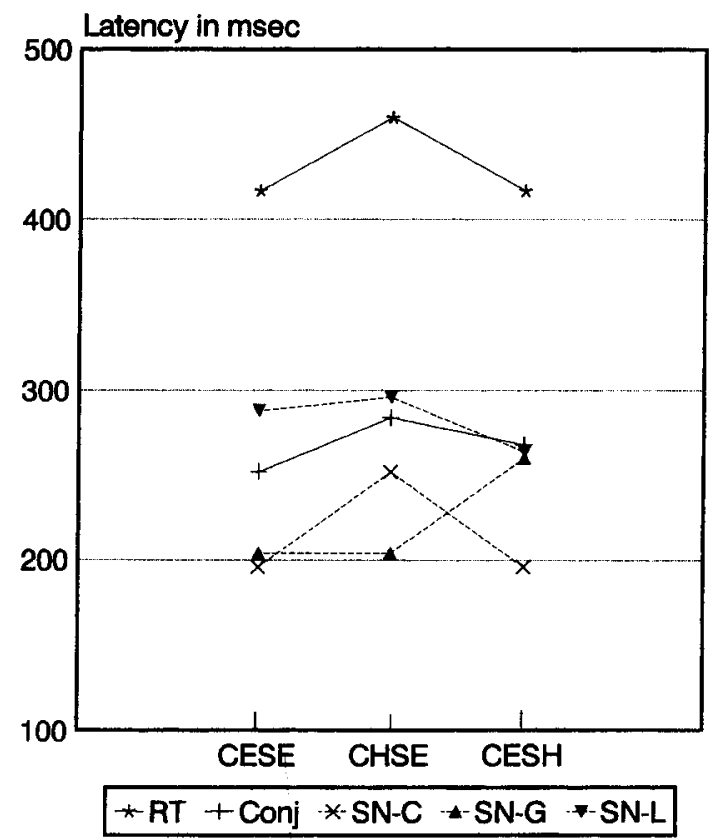

Figure 13. Averaged mean RTs and SN onset latencies in milliseconds for each of the three discriminability conditions. CESE denotes the latencies in the color-easy/shape-easy condition; CHSE denotes those in the color-hard/shape-easy condition; and CESH denotes those in the color-easy/shape-hard condition. Conj denotes the onset latency of conjunction-specific selective processing. SN-C, SN-G, and SN-L stand for the onset latencies of the SN related to, respectively, color selection, global shape selection, and local shape selection. 
If it does, it suggests dependent and self-terminating selections of the attributes (predictions of the selfterminating model in Table 1). If it does not, it suggests independent and exhaustive selections (exhaustive model, Table 1). If there are SNs larger than zero to one attribute at both levels of the other attribute and these start to differ as a function of the relevance of the latter, they suggest initially independent and exhaustive processing of the attributes followed by conjunction-specific processing (exhaustive model, Predictions 5-8, Table 1 ). If there is an SN only to the relevant conjunction (e.g., $\mathrm{C}+\mathrm{S}+$ ) stimulus, while the SNs to stimuli with one or more irrelevant attribute values (e.g., $C+S-, C-S++, C-S+$, $C-S-$ ) are zero, it suggests selection on the basis of a unique representation of the relevant conjunction (holistic model, Table 1).

In the CESE and CHSE conditions, the evidence disconfirmed the self-terminating and holistic predictions and confirmed those of the exhaustive model. We found main effects of color and global shape relevance that were initially additive, after which they started to interact. This means that Predictions $1-8$ of the exhaustive model were confirmed. In the CESE condition, Predictions 3 and 4 of the self-terminating model with equal identification times and Predictions 3 and 4 of the holistic model were disconfirmed. In the CHSE condition, Prediction 4 of the self-terminating model with unequal identification times and Predictions 3 and 4 of the holistic model were disconfirmed. In all three conditions, SNs related to local shape processing were found both when the color was relevant and when it was not. This falsifies Prediction 4 of the self-terminating model with unequal identification times. In the CESH condition, we found evidence for selfterminating processing of global shape when the color was irrelevant, violating Prediction 4 of the exhaustive model, and evidence for exhaustive processing of local shape, violating Prediction 4 of the self-terminating model.

That global shape was processed in a self-terminating fashion and local shape was processed exhaustively in CESH seems puzzling but can be explained if global and local shape are processed in parallel and as separable attributes (Pomerantz, 1983). In the CESH condition, the global shapes were very similar. For example, relevant global shapes were vertical parallel lines with different horizontal lines in between, and irrelevant global shapes were vertical parallel lines with different diagonal lines in between (a difference in orientation, see the introduction and Figure 1). In the circular shapes, there was a similar distinction: a gap on the vertical or on the horizontal meridian was relevant. Thus, global shape analysis consisted, for example, of discriminating between horizontal and diagonal line segments. Local shape analysis consisted of discriminating between two particular diagonal lines when "diagonal" was relevant. The present finding that local shape was selected on $C$ - trials, whereas global shape was not, suggests that the participants did not discriminate horizontal versus diagonal shape segments on these trials, but they did discriminate between diagonal lines. On $C+$ trials, both discriminations were made. Thus, the local shape discrimination could be made independent of global shape discrimination, which suggests separability. That, in the CESH condition, the onsets of the global and local shape $\mathrm{SN}$ effects were equal suggests that these discriminations took equally long and occurred in parallel (e.g., analyses of different shape features at the same spatial level).

Together, the findings lead to the conclusion that conjunctions of easy-to-discriminate colors and shapes are initially analyzed independently, exhaustively, and in parallel, after which processing becomes selective to the relevant conjunction. The findings thus support two-stage models of multidimensional selection in which the attributes of an object are processed in parallel by separate dimensional analyzers in a first stage, after which the outcomes of the analyses are integrated to form a unified percept of the object (e.g., Hoffman, 1979; Treisman \& Gelade, 1980).

The third issue addressed in the present study concerned the temporal organization of the two stages in this type of model. Does feature integration start only after the separate attributes have been identified, or can it start before identification of the separate attributes has finished? We found that, in the CESE condition, the onset latency of the SN related to local shape selection $(290 \mathrm{msec})$ was longer than the onset latency of the interaction between color relevance and global shape relevance (250 msec). In CHSE and CESH, the onset latency of the local shape SN was a little longer than or equal to the onset latency of the interaction (in CHSE, 300 and $280 \mathrm{msec}$, respectively; in CESH, 260 and $270 \mathrm{msec}$, respectively). Furthermore, the onset latency of the local shape $\mathrm{SN}$ was not delayed by the discriminability manipulations, but the onset of the color relevance $\times$ global shape relevance interaction was. These findings suggest that feature integration of color and global shape started before the local shape was available, and that feature integration and Stage 1 analysis of local shape were not performed in series. If these analyses had been performed in series, the onset of the SN related to local shape selection should have been delayed due to decreased discriminability. These results, therefore, suggest a model in which the second stage of feature integration can start before the first stage of independent and parallel processing of attributes has finished. Similar findings were obtained by Woods and Alain (1993) with an auditory selective attention task, which suggests that temporal overlap of separate attribute analyses and feature integration may be a general temporal organization of Stage 1 and Stage 2 processing.

That feature integration can start before all of the separate attribute analyses are finished raises questions about the transmission of information from Stage 1 to Stage 2. One question is whether the output of the separate attribute analyses are transmitted in a continuous, or in a discrete manner (cf. Miller, 1988). For example, Stage 1 may transmit preliminary output consisting of continuously varying activation levels of feature nodes in a neural network. Alternatively, it may consist of the end result of each attribute's analysis, represented as a discrete psycho- 
logical code (cf. Posner, 1978). The present data seem to favor the latter alternative because, in all three discriminability conditions, conjunction-specific processing started only after both color and global shape relevance had already started to produce additive ERP differences for $50 \mathrm{msec}$. If Stage 1 would transmit output in a continuous manner to Stage 2 , the color $\times$ shape interactions should have started at the same time as the main effects of color and shape, because a little bit of available color and shape information would have already started the feature integration process.

\section{REFERENCES}

Arguin, M., \& Cavanagh, P. (1988). Parallel processing of two disjunctive targets. Perception \& Psychophysics, 44, 22-30.

Biederman, I., \& Checkosky, S. F. (1970). Processing redundant information. Journal of Experimental Psychology, 83, 486-490.

Desimone, R., \& UnGerLeider, L. G. (1989). Neural mechanisms of visual processing in monkeys. In F. Boller \& J. Grafman (Eds.), Handbook of neuropsychology (Vol. 2, pp. 267-299). Amsterdam: Elsevier.

EGETH, H. E. (1966). Parallel versus serial processes in multidimensional stimulus discrimination. Perception \& Psychophysics, 1, 245-252.

Ellis, S. H., \& CHASE, W. G. (1971). Parallel processing in item recognition. Perception \& Psychophysics, 10, 379-384.

Felfoldy, G. L., \& GarNeR, W. R. (1971). The effects on speeded classification of implicit and explicit instructions regarding redundant dimensions. Perception \& Psychophysics, 9, 289-292.

GARNER, W. R. (1974). The processing of information and structure. Potomac, MD: Erlbaum.

GARNER, W. R. (1987). Location and color as cuing dimensions in contingent classification. Perception \& Psychophysics, 41, 202-210.

GRILL, D. P. (1971). Variables influencing the mode of processing of complex stimuli. Perception \& Psychophysics, 10, 51-57.

Guthrie, D., \& Buchwald, J. S. (1991). Significance testing of difference potentials. Psychophysiology, 28, 240-244.

HanSEN, J. C., \& Hillyard, S. A. (1983). Selective attention to multidimensional auditory stimuli. Journal of Experimental Psychology: Human Perception \& Performance, 9, 1-19.

HARTER, M. R., \& AINE, C. J. (1984). Brain mechanisms of visual selective attention. In R. Parasuraman \& D. R. Davies (Eds.), Varieties of attention (pp. 293-321). Orlando, FL: Academic Press.

HARTER, M. R., \& AINE, C. J. (1986). Discussion of the neural-specificity model of selective attention: A response to Hillyard \& Mangun and Näätänen. Biological Psychology, 23, 297-312.

Hawkins, H. L. (1969). Parallel processing in complex visual discrimination. Perception \& Psychophysics, 5, 56-64.

Hillyard, S. A., \& MangUN, G. R. (1986). The neural basis of visual selective attention: A commentary on Harter and Aine. Biological Psychology, 23, 265-279.

Hillyard, S. A., \& MüNTE, T. F. (1984). Selective attention to color and location: An analysis with event-related brain potentials. Perception \& Psychophysics, 36, 185-198.

Hillyard, S. A., \& Picton, T. W. (1987). Electrophysiology of cognition. In F. Plum (Ed.), Handbook of physiology: Sec. I. The nervous system: Vol. 5. Higher function of the nervous system, Part 2 (pp. $519-$ 584). Baltimore, MD: American Physiological Society.

Hoffman, J. E. (1979). A two-stage model of visual search. Perception \& Psychophysics, 25, 319-327.

Kenemans, J. L., KoK, A., \& Smulders, F. T. Y. (1993). Event-related potentials to conjunctions of spatial frequency and orientation as a function of stimulus parameters and response requirements. Electroencephalography \& Clinical Neurophysiology, 88, 51-63.

KIMCHI, R. (1992). Primacy of wholistic processing and global/local paradigm: A critical review. Psychological Bulletin, 112, 24-38.

KuTAS, M., \& Donchin, E. (1980). Preparation to respond as manifested by movement-related brain potentials. Brain Research, 202, 95-115.
MANGUn, G. R., \& Hillyard, S. A. (1995). Mechanisms and models of selective attention. In M. D. Rugg \& M. G. H. Coles (Eds.), Electrophysiology of mind: Event-related potentials and cognition (pp. 86131). New York: Oxford University Press.

McClean, J. P., Broadbent, D. E., \& Broadbent, M. H. P. (1982). Combining attributes in rapid sequential visual presentation. Quarterly Journal of Experimental Psychology, 35A, 171-186.

Miller, J. O. (1988). Discrete and continuous models of human information processing: Theoretical distinctions and empirical results. Acta Psychologica, 67, 191-257.

MORDKOFF, J. T., \& YANTIS, S. (1993). Dividing attention between color and shape: Evidence of coactivation. Perception \& Psychophysics, 53, 357-366.

NÄÄTÄNEN, R. (1992). Attention and brain function. Hillsdale, NJ: Erlbaum.

Pomerantz, J. R. (1983). Global and local precedence: Selective attention in form and motion perception. Journal of Experimental Psychology: General, 112, 516-540.

Posner, M. (1978). Chronometric explorations of mind. Hillsdale, $\mathrm{NJ}$ : Erlbaum.

RUGG, M. D. (1991). ERPs and selective attention: Commentary. Electroencephalography \& Clinical Neurophysiology (Suppl. 42), 222-227.

Saraga, E., \& Shallice, T. (1973). Parallel processing of the attributes of single stimuli. Perception \& Psychophysics, 13, 261-270.

SCHERG, M. (1992). Brain electrical source analysis: Version 1.9 [Computer software]. Munich: Scherg \& Berg.

Smid, H. G. O. M., Böcker, K. B. E., van Touw, D. A., Muldé, G., \& BRUNIA, C. H. M. (1996). A psychophysiological investigation of the selection and use of partial stimulus information in response choice. Journal of Experimental Psychology: Human Perception \& Performance, 22, 3-24.

Smid, H. G. O. M., Mulder, G., Mulder, L. J. M., \& Brands, G. J. (1992). A psychophysiological study of the use of partial information in stimulus response translation. Journal of Experimental Psychology: Human Perception \& Performance, 18, 1101-1119.

Snodgrass, J. G., \& Townsend, J. T. (1980). Comparing parallel and serial models: Theory and implementation. Journal of Experimental Psychology: Human Perception \& Performance, 6, 330-354.

Townsend, J. T., \& AshBy, F. G. (1983). Stochastic modeling of elementary psychological processes. Cambridge: Cambridge University Press.

Treisman, A. [M.] (1993). The perception of features and objects. In A. [D.] Baddeley \& L. Weiskrantz (Eds.), Attention: Selection, awareness, and control. Oxford: Oxford University Press, Clarendon Press.

Treisman, A. M., \& Gelade, G. (1980). A feature-integration theory of attention. Cognitive Psychology, 12, 97-136.

Treisman, A. M., \& Paterson, R. (1984). Emergent features, attention and object perception. Journal of Experimental Psychology: Human Perception \& Performance, 10, 12-31.

van der heidden, A. H. C., La Heis, W., Phaf, R. H., Buijs, D. A. C. H., \& VAN VLIET, E. C. (1988). Response competition and condition competition in visual selective attention. Acta Psychologica, 67, 259-277.

WATANABE, T. (1988). Effect of irrelevant differences as a function of the relations between relevant and irrelevant dimensions in the samedifferent task. Journal of Experimental Psychology: Human Perception \& Performance, 14, 132-142.

WIJERs, A. A. (1989). Visual selective attention: An electrophysiological approach. Unpublished doctoral dissertation, University of Groningen. Wijers, A. A., Lamain, W., Slopsema, S., Mulder, G., \& Mulder, L. J. M. (1989). An electrophysiological investigation of the spatial distribution of attention to colored stimuli in focussed and divided attention conditions. Biological Psychology, 29, 213-245.

Wijers, A. A., Mulder, G., Okita, T., \& Mulder, L. J. M. (1989). Event-related potentials during memory scarch and selective attention to letter size and conjunction of letter size and color. Psychophysiology, 26, 529-547.

Woods, D. L., \& Alain, C. (1993). Feature processing during high-rate auditory selective attention. Perception \& Psychophysics, 53, 391-402.

WoOds, D. L., Alho, K., \& AlgazI, A. (1994). Stages of auditory feature conjunction: An event-related brain potential study. Journal of Experimental Psychology: Human Perception \& Performance, 20, 81-94. 


\section{NOTES}

1. It is important to note that, because the $\mathrm{SN}$ is a relative measure (it is a difference potential), its presence does not mean that relevant stimuli received processing and irrelevant stimuli did not. From its presence, it can be inferred only that relevant stimuli received more processing than did irrelevant stimuli. Furthermore, the absence of an SN in the ERPs to relevant and irrelevant attribute values would not imply that the attribute was not identified.

2. Note that this is equivalent to the formulation used by Woods et al. (1994). These authors define the time that conjunction-specific processing occurs as the time at which the total effect of relevance-that is, the difference between $[\operatorname{ERP}(C+S+)-\operatorname{ERP}(C-S-)]$-is larger than the sum of the separate effects of relevance of the attributes - that is, the sum of the differences $[\operatorname{ERP}(C+S-)-\operatorname{ERP}(C-S-)]$ and $[\operatorname{ERP}(C-S+)-$ $\operatorname{ERP}(C-S-)]$

3. Note that the predictions concerning the serial/parallel and selfterminating/exhaustive issues, as formulated in the present approach, do not depend on specific interpretations of the cognitive correlate of the $\mathrm{SN}$. As long as the $\mathrm{SN}$ can be assumed to be an index of selective processing, most hypotheses regarding its cognitive correlates would lead to the same conclusions with regard to these issues.

4. In the preliminary experiment, we adjusted the relative discriminability of the colors and the shapes of the stimuli in the easy- and harddiscrimination conditions. Six participants performed four disjunctive choice RT discrimination tasks, in which they responded with one hand to one value on a dimension and with the other hand to the other value on that dimension (see Figure 2 for the stimuli used in these tasks). In the color-easy task, the participants had to discriminate the red and blue colors of the CESE stimuli, while ignoring the variations in shape $(\mathrm{RT}=342 \mathrm{msec})$. In the shape-easy task, the participants had to discriminate the global shape configurations of the CESE stimuli (i.e., the closure and the parallel-line stimuli), ignoring their red and blue color variations and local shape variations ( $R T=355 \mathrm{msec}$ ). In the color-hard task, they had to discriminate light-red and red CHSE stimuli in some blocks of trials ( $R T=386 \mathrm{msec}$ ) and light-blue and blue CHSE stimuli in other blocks ( $R T=394 \mathrm{msec}$ ). In the shape-hard condition, they had to discriminate local shape segments within each global configuration. For example, in some blocks, all stimuli were closure shapes (the CESH $B$ stimuli in Figure 2), and the participants had to respond with the left hand to circles having a gap in their left or right side and with the right hand to circles having a gap in their upper or lower part $(\mathrm{RT}=\mathbf{4 0 2} \mathrm{msec})$. In other blocks, all stimuli were parallel-line configurations (the CESH $A$ stimuli), and the participants responded with the left hand to parallel lines with a horizontal line segment and with the right hand to parallel lines with a diagonal line segment $(\mathrm{RT}=412 \mathrm{msec})$. It is important to note that decreasing the discriminability of shape involved the discriminability of global shape and not the discriminability of local shape. The RTs show that there was a substantial increase in disjunctive RT when an attribute was made more difficult to discriminate $(50 \mathrm{msec})$, and this increase was the same for the colors and the shapes. There was no significant difference between color classification RTs and shape classification RTs. Within-subject repeated measures ANOVAs with the factors attribute (color and shape) and discriminability (easy and hard) support these interpretations. The RTs significantly increased with decreasing discriminability $\left[F(1,5)=137.17, M S_{\mathrm{e}}=345, p<.0005\right]$ and did not differ whether the relevant attribute was color or shape $[F(1,5)=3.82$, $\left.M S_{\mathrm{e}}=345, p>.10\right]$. These factors did not interact $\left[F(1,5)<1, M S_{\mathrm{e}}=\right.$ 218]. The RTs to hard- and easy-to-discriminate colors differed significantly $\left[F(1,5)=27.90, M S_{\mathrm{e}}=243, p<.003\right]$, as well as did those to hard- and easy-to-discriminate shapes $\left[F(1,5)=96.52, M S_{\mathrm{e}}=84, p<\right.$ $.0005]$. Error rates did not differ between easy and hard conditions (smallest $p=.086$ ), ruling out speed-accuracy effects. These results were replicated with 8 other participants, who made go/no-go responses to discriminate the same values of the attributes. Thus, in terms of disjunctive discrimination RT, the relative discriminability of the "easy" colors was equivalent to that of the "easy" shapes, and the relative discriminability of the "hard" colors was equivalent to that of the "hard" shapes.

(Manuscript received November 6, 1995; revision accepted for publication July 21,1996 .) 